\title{
Invariant grids for reaction kinetics
}

\author{
Alexander N. Gorban \\ Department of Materials, Institute of Polymer Physics \\ Swiss Federal Institute of Technology, CH-8092 Zürich, Switzerland \\ Institute of Computational Modeling RAS, Krasnoyarsk 660036, Russia \\ Iliya V. Karlin \\ Department of Materials, Institute of Polymer Physics \\ Swiss Federal Institute of Technology, CH-8092 Zürich, Switzerland \\ Andrei Yu. Zinovyev \\ Institut des Hautes Etudes Scientifiques, \\ Le Bois-Marie, 35, route de Chartres, F-91440, Bures-sur-Yvette, France
}

\begin{abstract}
In this paper, we review the construction of low-dimensional manifolds of reduced description for equations of chemical kinetics from the standpoint of the method of invariant manifold (MIM). MIM is based on a formulation of the condition of invariance as an equation, and its solution by Newton iterations. A grid-based version of MIM is developed. Generalizations to open systems are suggested. The set of methods covered makes it possible to effectively reduce description in chemical kinetics.

The most essential new element of this paper is the systematic consideration of a discrete analogue of the slow (stable) positively invariant manifolds for dissipative systems, invariant grids. We describe the Newton method and the relaxation method for the invariant grids construction. The problem of the grid correction is fully decomposed into the problems of the grid's nodes correction. The edges between the nodes appears only in the calculation of the tangent spaces. This fact determines high computational efficiency of the invariant grids method.
\end{abstract}

Key words: Kinetics, Model Reduction, Grids, Invariant Manifold, Entropy, Nonlinear Dynamics, Mathematical Modeling

Email addresses: agorban@mat.ethz.ch (Alexander N. Gorban), ikarlin@mat.ethz.ch (Iliya V. Karlin), zinovyev@ihes.fr (Andrei Yu. Zinovyev). 


\section{Contents}

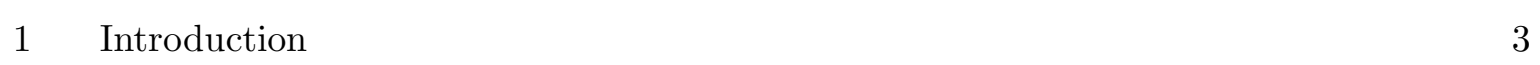

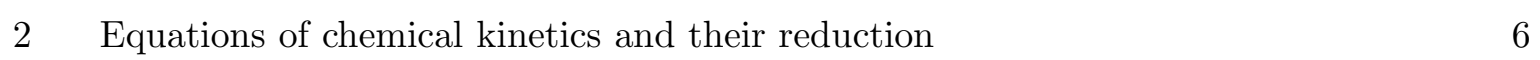

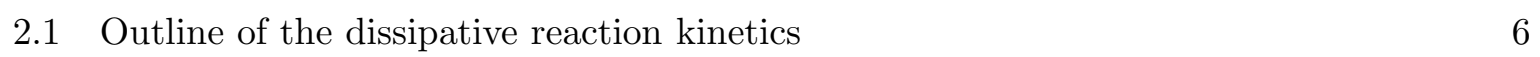

2.2 The problem of reduced description in chemical kinetics 9

$\begin{array}{lll}2.3 & \text { Partial equilibrium approximations } & 10\end{array}$

$\begin{array}{lll}2.4 \text { Model equations } & 12\end{array}$

$\begin{array}{lll}2.5 & \text { Quasi-steadv state approximation } & 13\end{array}$

2.6 Methods based on spectral decomposition of Jacobian fields 15

2.7 Thermodvnamic criteria for selection of important reactions 19

$3 \quad$ Outline of the method of invariant manifold 20

$4 \quad$ Thermodvnamic projector 21

4.1 Thermodvnamic parameterization 21

4.2 Decomposition of motions: Thermodvnamics 23

$\begin{array}{lll}5 & \text { Corrections } & 23\end{array}$

5.1 Preliminary discussion 23

$\begin{array}{lll}5.2 & \text { Svmmetric linearization } & 25\end{array}$

5.3 Decomposition of motions: Kinetics 26

$\begin{array}{lll}5.4 \quad \text { Svmmetric iteration } & 27\end{array}$

$6 \quad$ The method of invariant manifold 27

$7 \quad$ Illustration: Two-step catalvtic reaction 28

$8 \quad$ Relaxation methods 31

$9 \quad$ Method of invariant manifold without a priori parameterization $\quad 31$

$10 \quad$ Method of invariant grids 33

10.1 Grid construction strategy 36

\begin{tabular}{|ll}
10.2 Instability of fine grids & 37
\end{tabular} 
10.3 What space is the most appropriate for the grid construction?

10.4 Carleman's formulas in the analvtical invariant manifolds approximations. First profit from analvtic

10.5 Example: Two-step catalvtic reaction 41

10.6 Example: Model hvdrogen burning reaction 43

11 Method of invariant manifold for open svstems 46

\begin{tabular}{lll}
\hline 12 Conclusion & 47
\end{tabular}

$\begin{array}{ll}\text { References } & 48\end{array}$

\section{Introduction}

In this paper, we present a general method of constructing the reduced description for dissipative systems of reaction kinetics and a new method of invariant grids. Our approach is based on the method of invariant manifold which was developed in end of 1980th - beginning of 1990th $[24,25,26]$. Its realization for a generic dissipative systems was discussed in $[28,36]$. This method was applied to a set of problems of classical kinetic theory based on the Boltzmann kinetic equation $[28,49,51]$. The method of invariant manifold was successfully applied to a derivation of reduced description for kinetic equations of polymeric solutions [79]. It was also been tested on systems of chemical kinetics [35,32]. In order to construct manifolds of a relatively low dimension, grid-based representations of manifolds become a relevant option. The idea of invariant grids was suggested recently in [32].

The goal of nonequilibrium statistical physics is the understanding of how a system with many degrees of freedom acquires a description with a few degrees of freedom. This should lead to reliable methods of extracting the macroscopic description from a detailed microscopic description.

Meanwhile this general problem is still far from the final solution, it is reasonable to study simplified models, where, on the one hand, a detailed description is accessible to numerics, on the other hand, analytical methods designed to the solution of problems in real systems can be tested.

In this paper we address the well known class of finite-dimensional systems known from the theory of reaction kinetics. These are equations governing a complex relaxation in perfectly stirred closed chemically active mixtures. Dissipative properties of such systems are characterized with a global convex Lyapunov function $G$ (thermodynamic potential) which implements the second law of thermodynamics: As the time $t$ tends to infinity, the system reaches the unique equilibrium state while in the course of the transition the Lyapunov function decreases monotonically.

While the limiting behavior of the dissipative systems just described is certainly very 
simple, there are still interesting questions to be asked about. One of these questions is closely related to the above general problem of nonequilibrium statistical physics. Indeed, evidence of numerical integration of such systems often demonstrates that the relaxation has a certain geometrical structure in the phase space. Namely, typical individual trajectories tend to manifolds of lower dimension, and further proceed to the equilibrium essentially along these manifolds. Thus, such systems demonstrate a dimensional reduction, and therefore establish a more macroscopic description after some time since the beginning of the relaxation.

There are two intuitive ideas behind our approach, and we shall now discuss them informally. Objects to be considered below are manifolds (surfaces) $\boldsymbol{\Omega}$ in the phase space of the reaction kinetic system (the phase space is usually a convex polytope in a finitedimensional real space). The 'ideal' picture of the reduced description we have in mind is as follows: A typical phase trajectory, $\boldsymbol{c}(t)$, where $t$ is the time, and $\boldsymbol{c}$ is an element of the phase space, consists of two pronounced segments. The first segment connects the beginning of the trajectory, $\boldsymbol{c}(0)$, with a certain point, $\boldsymbol{c}\left(t_{1}\right)$, on the manifold $\Omega$ (rigorously speaking, we should think of $\boldsymbol{c}\left(t_{1}\right)$ not on $\Omega$ but in a small neighborhood of $\Omega$ but this is inessential for the ideal picture). The second segment belongs to $\Omega$, and connects the point $\boldsymbol{c}\left(t_{1}\right)$ with the equilibrium $\boldsymbol{c}^{\mathrm{eq}}=\boldsymbol{c}(\infty), \boldsymbol{c}^{\mathrm{eq}} \in \Omega$. Thus, the manifolds appearing in our ideal picture are "patterns" formed by the segments of individual trajectories, and the goal of the reduced description is to "filter out" this manifold.

There are two important features behind this ideal picture. The first feature is the invariance of the manifold $\Omega$ : Once the individual trajectory has started on $\Omega$, it does not leaves $\Omega$ anymore. The second feature is the projecting: The phase points outside $\Omega$ will be projected onto $\Omega$. Furthermore, the dissipativity of the system provides an additional information about this ideal picture: Regardless of what happens on the manifold $\Omega$, the function $G$ was decreasing along each individual trajectory before it reached $\Omega$. This ideal picture is the guide to extract slow invariant manifolds.

One more point needs a clarification before going any further. Low dimensional invariant manifolds exist also for systems with a more complicated dynamic behavior, so why to study the invariant manifolds of slow motions for a particular class of purely dissipative systems? The answer is in the following: Most of the physically significant models include non-dissipative components in a form of either a conservative dynamics, or in the form of external forcing or external fluxes. Example of the first kind is the free flight of particles on top of the dissipation-producing collisions in the Boltzmann equation. For the second type of example one can think of irreversible reactions among the suggested stoichiometric mechanism (inverse process are so unprobable that we discard them completely thereby effectively "opening" the system to the remaining irreversible flux). For all such systems, the present method is applicable almost without special refinements, and bears the significance that invariant manifolds are constructed as a "deformation" of the relevant manifolds of slow motion of the purely dissipative dynamics. Example of this construction for open systems is presented below in section 11. Till then we focus on the purely dissipative case for the reason just clarified.

The most essential new element of this paper is the systematic consideration of a dis- 
crete analogue of the slow (stable) positively invariant manifolds for dissipative systems, invariant grids. These invariant grids were introduced in the [32]. Here we will describe the Newton method subject to incomplete linearization and the relaxation methods for the invariant grids. It is worth to mention, that the problem of the grid correction is fully decomposed into the problems of the grid's nodes correction. The edges between the nodes appears only in the calculation of the tangent spaces. This fact determines high computational efficiency of the invariant grids method.

Due to the famous Lyapunov auxiliary theorem $[60,54]$ we can construct analytical invariant manifolds for kinetic equations with analytical right hand side. Moreover, the analycity can serve as a "selection rule" for selection the unique analytic positively invariant manifold from the infinite set of smooth positively invariant manifolds. The analycity gives a possibility to use the powerful technique of analytical continuation and Carleman's formulae $[1,38,39]$. It leads us to superresolution effects: A small grid may be sufficient to present an "large" analytical manifold immersed in the whole space.

The paper is organized as follows. In the section 2, we review the reaction kinetics (section 2.1), and discuss the main methods of model reduction in chemical kinetics (section 2.2). In particular, we present two general versions of extending partially equilibrium manifolds to a single relaxation time model in the whole phase space, and develop a thermodynamically consistent version of the intrinsic low-dimensional manifold (ILDM) approach. In the section 3 we review the method of invariant manifold in the way appropriate to this class of nonequilibrium systems. In the sections 4 and 5 we give some details on the two relatively independent parts of the method, the thermodynamic projector, and the iterations for solving the invariance equation.

We also describe a general symmetric linearization procedure for the invariance equation, and discuss its relevance to the picture of decomposition of motions. In the section 6 , these two procedures are combined into an unique algorithm. In the section 7 , we demonstrate an illustrative example of analytic computations for a model catalytic reaction. In the section 8 we introduce the relaxation method for solution the invariance equation. This relaxation method is an alternative to the Newton iteration method. In the section 9 we demonstrate how the thermodynamic projector is constructed without the a priori parameterization of the manifold ${ }^{1}$. This result is essentially used in the section 10 where we introduce a computationally effective grid-based method to construct invariant manifolds. It is the central section of the paper. We present the Newton method and the relaxation method for the grid construction. The Carleman formulas for analytical continuation a manifold from a grid are proposed. Two examples of kinetic equations are analyzed: a two-dimensional catalytic reaction (four species, two balances) and a four-dimensional oxidation reaction (six species, two balances).

In the section 11 we describe an extension of the method of invariant manifold to open

1 This thermodynamic projector is the unique operator which transforms the arbitrary vector field equipped with the given Lyapunov function into a vector field with the same Lyapunov function (and also this happens on any manifold which is not tangent to the level of the Lyapunov function). 
systems. Finally, results are discussed in the section 12 .

\section{Equations of chemical kinetics and their reduction}

\subsection{Outline of the dissipative reaction kinetics}

We begin with an outline of the reaction kinetics (for details see e. g. the book of [77]). Let us consider a closed system with $n$ chemical species $\mathrm{A}_{1}, \ldots, \mathrm{A}_{n}$, participating in a complex reaction. The complex reaction is represented by the following stoichiometric mechanism:

$$
\alpha_{s 1} \mathrm{~A}_{1}+\ldots+\alpha_{s n} \mathrm{~A}_{n} \rightleftharpoons \beta_{s 1} \mathrm{~A}_{1}+\ldots+\beta_{s n} \mathrm{~A}_{n}
$$

where the index $s=1, \ldots, r$ enumerates the reaction steps, and where integers, $\alpha_{s i}$ and $\beta_{s i}$, are stoichiometric coefficients. For each reaction step $s$, we introduce $n$-component vectors $\boldsymbol{\alpha}_{s}$ and $\boldsymbol{\beta}_{s}$ with components $\alpha_{s i}$ and $\beta_{s i}$. Notation $\boldsymbol{\gamma}_{s}$ stands for the vector with integer components $\gamma_{s i}=\beta_{s i}-\alpha_{s i}$ (the stoichiometric vector). We adopt an abbreviated notation for the standard scalar product of the $n$-component vectors:

$$
(\boldsymbol{x}, \boldsymbol{y})=\sum_{i=1}^{n} x_{i} y_{i}
$$

The system is described by the $n$-component concentration vector $\boldsymbol{c}$, where the component $c_{i} \geq 0$ represents the concentration of the specie $\mathrm{A}_{i}$. Conservation laws impose linear constraints on admissible vectors $\boldsymbol{c}$ (balances):

$$
\left(\boldsymbol{b}_{i}, \boldsymbol{c}\right)=B_{i}, i=1, \ldots, l
$$

where $\boldsymbol{b}_{i}$ are fixed and linearly independent vectors, and $B_{i}$ are given scalars. Let us denote as $\boldsymbol{B}$ the set of vectors which satisfy the conservation laws (2):

$$
\boldsymbol{B}=\left\{\boldsymbol{c} \mid\left(\boldsymbol{b}_{1}, \boldsymbol{c}\right)=B_{1}, \ldots,\left(\boldsymbol{b}_{l}, \boldsymbol{c}\right)=B_{l}\right\}
$$

The phase space $\boldsymbol{V}$ of the system is the intersection of the cone of $n$-dimensional vectors with nonnegative components, with the set $\boldsymbol{B}$, and $\operatorname{dim} \boldsymbol{V}=d=n-l$. In the sequel, we term a vector $\boldsymbol{c} \in \boldsymbol{V}$ the state of the system. In addition, we assume that each of the conservation laws is supported by each elementary reaction step, that is

$$
\left(\boldsymbol{\gamma}_{s}, \boldsymbol{b}_{i}\right)=0
$$

for each pair of vectors $\boldsymbol{\gamma}_{s}$ and $\boldsymbol{b}_{i}$. 
Reaction kinetic equations describe variations of the states in time. Given the stoichiometric mechanism (1), the reaction kinetic equations read:

$$
\dot{\boldsymbol{c}}=\boldsymbol{J}(\boldsymbol{c}), \boldsymbol{J}(\boldsymbol{c})=\sum_{s=1}^{r} \gamma_{s} W_{s}(\boldsymbol{c}),
$$

where dot denotes the time derivative, and $W_{s}$ is the reaction rate function of the step $s$. In particular, the mass action law suggests the polynomial form of the reaction rates:

$$
W_{s}=k_{s}^{+} \prod_{i=1}^{n} c_{i}^{\alpha_{i}}-k_{s}^{-} \prod_{i=1}^{n} c_{i}^{\beta_{i}},
$$

where $k_{s}^{+}$and $k_{s}^{-}$are the constants of the direct and of the inverse reactions rates of the $s$ th reaction step. The phase space $\boldsymbol{V}$ is positive-invariant of the system (4): If $\boldsymbol{c}(0) \in \boldsymbol{V}$, then $\boldsymbol{c}(t) \in \boldsymbol{V}$ for all the times $t>0$.

In the sequel, we assume that the kinetic equation (4) describes evolution towards the unique equilibrium state, $\boldsymbol{c}^{\mathrm{eq}}$, in the interior of the phase space $\boldsymbol{V}$. Furthermore, we assume that there exists a strictly convex function $G(\boldsymbol{c})$ which decreases monotonically in time due to Eq. (4) ${ }^{2}$ :

$$
\dot{G}=(\nabla G(\boldsymbol{c}), \boldsymbol{J}(\boldsymbol{c})) \leq 0
$$

Here $\nabla G$ is the vector of partial derivatives $\partial G / \partial c_{i}$, and the convexity assumes that the $n \times n$ matrices

$$
\boldsymbol{H}_{\boldsymbol{c}}=\left\|\partial^{2} G(\boldsymbol{c}) / \partial c_{i} \partial c_{j}\right\|
$$

are positive definite for all $\boldsymbol{c} \in \boldsymbol{V}$. In addition, we assume that the matrices (7) are invertible if $\boldsymbol{c}$ is taken in the interior of the phase space.

The function $G$ is the Lyapunov function of the system (4), and $\boldsymbol{c}^{\mathrm{eq}}$ is the point of global minimum of the function $G$ in the phase space $\boldsymbol{V}$. Otherwise stated, the manifold of equilibrium states $\boldsymbol{c}^{\mathrm{eq}}\left(B_{1}, \ldots, B_{l}\right)$ is the solution to the variational problem,

$$
G \rightarrow \min \text { for }\left(\boldsymbol{b}_{i}, \boldsymbol{c}\right)=B_{i}, i=1, \ldots, l .
$$

$\overline{2}$ With some abuse of language, we can term the functional $-G$ the entropy, although it is a different functional for non-isolated systems. We recall that thermodynamic Lyapunov functions are well defined not just for isolated systems. Such functionals are easily constructed also for systems which exchange energy and/or matter with a larger equilibrium system (with a thermostat, for example). In such a case, the thermodynamic Lyapunov function is constructed as the entropy of the minimal closed system containing the system under consideration [23]. In particular, the free energy and the free enthalpy (the Gibbs and the Helmholz energies, respectively) can be constructed in this manner. They are are identical with the entropy of the minimal closed system containing the given system within the accuracy of multiplication with a factor which remains constant in time, and subtracting a constant. 
For each fixed value of the conserved quantities $B_{i}$, the solution is unique. In many cases, however, it is convenient to consider the whole equilibrium manifold, keeping the conserved quantities as parameters.

For example, for perfect systems in a constant volume under a constant temperature, the Lyapunov function $G$ reads:

$$
G=\sum_{i=1}^{n} c_{i}\left[\ln \left(c_{i} / c_{i}^{\mathrm{eq}}\right)-1\right]
$$

It is important to stress that $\boldsymbol{c}^{\mathrm{eq}}$ in Eq. (9) is an arbitrary equilibrium of the system, under arbitrary values of the balances. In order to compute $G(\boldsymbol{c})$, it is unnecessary to calculate the specific equilibrium $\boldsymbol{c}^{\mathrm{eq}}$ which corresponds to the initial state $\boldsymbol{c}$. Moreover, for ideal systems, function $G$ is constructed from the thermodynamic data of individual species, and, as the result of this construction, it turns out that it has the form of Eq. (9). Let us mention here the classical formula for the free energy $F=R T V G$ :

$$
F=V R T \sum_{i=1}^{n} c_{i}\left[\left(\ln \left(c_{i} V_{\mathrm{Q}} i\right)-1\right)+F_{\text {int } i}(T)\right]
$$

where $V$ is the volume of the system, $T$ is the temperature, $V_{\mathrm{Q} i}=N_{0}\left(2 \pi \hbar^{2} / m_{i} k T\right)^{3 / 2}$ is the quantum volume of one mole of the specie $A_{i}, N_{0}$ is the Avogadro number, $m_{i}$ is the mass of the molecule of $\mathrm{A}_{i}, R=k N_{0}$, and $F_{\text {int } i}(T)$ is the free energy of the internal degrees of freedom per mole of $\mathrm{A}_{i}$.

Finally, we recall an important generalization of the mass action law (5), known as the Marcelin-De Donder kinetic function. This generalization was developed in [20] based on ideas of the thermodynamic theory of affinity [18]. We use the kinetic function suggested in [11]. Within this approach, the functions $W_{s}$ are constructed as follows: For a given strictly convex function $G$, and for a given stoichiometric mechanism (1), we define the gain $(+)$ and the loss $(-)$ rates of the $s$ th step,

$$
W_{s}^{+}=\varphi_{s}^{+} \exp \left[\left(\nabla G, \boldsymbol{\alpha}_{s}\right)\right], \quad W_{s}^{-}=\varphi_{s}^{-} \exp \left[\left(\nabla G, \boldsymbol{\beta}_{s}\right)\right]
$$

where $\varphi_{s}^{ \pm}>0$ are kinetic factors. The Marcelin-De Donder kinetic function reads: $W_{s}=$ $W_{s}^{+}-W_{s}^{-}$, and the right hand side of the kinetic equation (4) becomes,

$$
\boldsymbol{J}=\sum_{s=1}^{r} \boldsymbol{\gamma}_{s}\left\{\varphi_{s}^{+} \exp \left[\left(\boldsymbol{\nabla} G, \boldsymbol{\alpha}_{s}\right)\right]-\varphi_{s}^{-} \exp \left[\left(\boldsymbol{\nabla} G, \boldsymbol{\beta}_{s}\right)\right]\right\}
$$

For the Marcelin-De Donder reaction rate (11), the dissipation inequality (6) reads:

$$
\dot{G}=\sum_{s=1}^{r}\left[\left(\boldsymbol{\nabla} G, \boldsymbol{\beta}_{s}\right)-\left(\boldsymbol{\nabla} G, \boldsymbol{\alpha}_{s}\right)\right]\left\{\varphi_{s}^{+} e^{\left(\boldsymbol{\nabla} G, \boldsymbol{\alpha}_{s}\right)}-\varphi_{s}^{-} e^{\left(\boldsymbol{\nabla} G, \boldsymbol{\beta}_{s}\right)}\right\} \leq 0 .
$$


The kinetic factors $\varphi_{s}^{ \pm}$should satisfy certain conditions in order to make valid the dissipation inequality (13). A well known sufficient condition is the detail balance:

$$
\varphi_{s}^{+}=\varphi_{s}^{-}
$$

other sufficient conditions are discussed in detail elsewhere $[77,23,46,47]$. For the function $G$ of the form (9), the Marcelin-De Donder equation casts into the more familiar mass action law form (5).

\subsection{The problem of reduced description in chemical kinetics}

What does it mean, "to reduce the description of a chemical system"? This means the following:

(1) To shorten the list of species. This, in turn, can be achieved in two ways:

(i) To eliminate inessential components from the list;

(ii) To lump some of the species into integrated components.

(2) To shorten the list of reactions. This also can be done in several ways:

(i) To eliminate inessential reactions, those which do not significantly influence the reaction process;

(ii) To assume that some of the reactions "have been already completed", and that the equilibrium has been reached along their paths (this leads to dimensional reduction because the rate constants of the "completed" reactions are not used thereafter, what one needs are equilibrium constants only).

(3) To decompose the motions into fast and slow, into independent (almost-independent) and slaved etc. As the result of such a decomposition, the system admits a study "in parts". After that, results of this study are combined into a joint picture. There are several approaches which fall into this category: The famous method of the quasisteady state (QSS), pioneered by Bodenstein and Semenov and explored in considerable detail by many authors, in particular, in $[10,14,68,21,66]$, and many others; the quasi-equilibrium approximation [62,23,74,21,46,47]; methods of sensitivity analysis $[64,56]$; methods based on the derivation of the so-called intrinsic low-dimensional manifolds (ILDM, as suggested in [61]). Our method of invariant manifold (MIM, $[24,25,26,28,35,36])$ also belongs to this kind of methods.

Why to reduce description in the times of supercomputers?

First, in order to gain understanding. In the process of reducing the description one is often able to extract the essential, and the mechanisms of the processes under study become more transparent. Second, if one is given the detailed description of the system, then one should be able also to solve the initial-value problem for this system. But what should one do in the case where the the system is representing just a point in a threedimensional flow? The problem of reduction becomes particularly important for modeling the spatially distributed physical and chemical processes. Third, without reducing the kinetic model, it is impossible to construct this model. This statement seems paradoxal 
only at the first glance: How come, the model is first simplified, and is constructed only after the simplification is done? However, in practice, the typical for a mathematician statement of the problem, (Let the system of differential equations be given, then ...) is rather rarely applicable in the chemical engineering science for detailed kinetics. Some reactions are known precisely, some other - only hypothetically. Some intermediate species are well studied, some others - not, it is not known much about them. Situation is even worse with the reaction rates. Quite on the contrary, the thermodynamic data (energies, enthalpies, entropies, chemical potentials etc) for sufficiently rarefied systems are quite reliable. Final identification of the model is always done on the basis of comparison with the experiment and with a help of fitting. For this purpose, it is extremely important to reduce the dimension of the system, and to reduce the number of tunable parameters. The normal logics of modeling for the purpose of chemical engineering science is the following: Exceedingly detailed but coarse with respect to parameters system $\rightarrow$ reduction $\rightarrow$ fitting $\rightarrow$ reduced model with specified parameters (cycles are allowed in this scheme, with returns from fitting to more detailed models etc). A more radical viewpoint is also possible: In the chemical engineering science, detailed kinetics is impossible, useless, and it does not exist. For a recently published discussion on this topic see $[57,58] ;[76]$.

Alas, with a mathematical statement of the problem related to reduction, we all have to begin with the usual: Let the system of differential equations be given ... . Enormous difficulties related to the question of how well the original system is modeling the real kinetics remain out of focus of these studies.

Our present work is devoted to studying reductions in a given system of kinetic equations to invariant manifolds of slow motions. We begin with a brief discussion of existing approaches.

\subsection{Partial equilibrium approximations}

Quasi-equilibrium with respect to reactions is constructed as follows: From the list of reactions (1), one selects those which are assumed to equilibrate first. Let they be indexed with the numbers $s_{1}, \ldots, s_{k}$. The quasi-equilibrium manifold is defined by the system of equations,

$$
W_{s_{i}}^{+}=W_{s_{i}}^{-}, i=1, \ldots, k
$$

This system of equations looks particularly elegant when written in terms of conjugated (dual) variables, $\boldsymbol{\mu}=\nabla G$ :

$$
\left(\boldsymbol{\gamma}_{s_{i}}, \boldsymbol{\mu}\right)=0, i=1, \ldots, k
$$

In terms of conjugated variables, the quasi-equilibrium manifold forms a linear subspace. This subspace, $L^{\perp}$, is the orthogonal completement to the linear envelope of vectors, $L=\operatorname{lin}\left\{\gamma_{s_{1}}, \ldots, \gamma_{s_{k}}\right\}$. 
Quasi-equilibrium with respect to species is constructed practically in the same way but without selecting the subset of reactions. For a given set of species, $A_{i_{1}}, \ldots, A_{i_{k}}$, one assumes that they evolve fast to equilibrium, and remain there. Formally, this means that in the $k$-dimensional subspace of the space of concentrations with the coordinates $c_{i_{1}}, \ldots, c_{i_{k}}$, one constructs the subspace $L$ which is defined by the balance equations, $\left(\boldsymbol{b}_{i}, \boldsymbol{c}\right)=0$. In terms of the conjugated variables, the quasi-equilibrium manifold, $L^{\perp}$, is defined by equations,

$$
\boldsymbol{\mu} \in L^{\perp},\left(\boldsymbol{\mu}=\left(\mu_{1}, \ldots, \mu_{n}\right)\right) .
$$

The same quasi-equilibrium manifold can be also defined with the help of fictitious reactions: Let $\boldsymbol{g}_{1}, \ldots, \boldsymbol{g}_{q}$ be a basis in $L$. Then Eq. (17) may be rewritten as follows:

$$
\left(\boldsymbol{g}_{i}, \boldsymbol{\mu}\right)=0, i=1, \ldots, q .
$$

Illustration: Quasi-equilibrium with respect to reactions in hydrogen oxidation: Let us assume equilibrium with respect to dissociation reactions, $\mathrm{H}_{2} \rightleftharpoons 2 \mathrm{H}$, and, $\mathrm{O}_{2} \rightleftharpoons 2 \mathrm{O}$, in some subdomain of reaction conditions. This gives:

$$
k_{1}^{+} c_{\mathrm{H}_{2}}=k_{1}^{-} c_{\mathrm{H}}^{2}, k_{2}^{+} c_{\mathrm{O}_{2}}=k_{2}^{-} c_{\mathrm{O}}^{2} .
$$

Quasi-equilibrium with respect to species: For the same reaction, let us assume equilibrium over $\mathrm{H}, \mathrm{O}, \mathrm{OH}$, and $\mathrm{H}_{2} \mathrm{O}_{2}$, in a subdomain of reaction conditions. Subspace $L$ is defined by balance constraints:

$$
c_{\mathrm{H}}+c_{\mathrm{OH}}+2 c_{\mathrm{H}_{2} \mathrm{O}_{2}}=0, c_{\mathrm{O}}+c_{\mathrm{OH}}+2 c_{\mathrm{H}_{2} \mathrm{O}_{2}}=0 .
$$

Subspace $L$ is two-dimensional. Its basis, $\left\{\boldsymbol{g}_{1}, \boldsymbol{g}_{2}\right\}$ in the coordinates $c_{\mathrm{H}}, c_{\mathrm{O}}, c_{\mathrm{OH}}$, and $c_{\mathrm{H}_{2} \mathrm{O}_{2}}$ reads:

$$
\boldsymbol{g}_{1}=(1,1,-1,0), \quad \boldsymbol{g}_{2}=(2,2,0,-1) .
$$

Corresponding Eq. (18) is:

$$
\mu_{\mathrm{H}}+\mu_{\mathrm{O}}=\mu_{\mathrm{OH}}, 2 \mu_{\mathrm{H}}+2 \mu_{\mathrm{O}}=\mu_{\mathrm{H}_{2} \mathrm{O}_{2}} .
$$

General construction of the quasi-equilibrium manifold: In the space of concentration, one defines a subspace $L$ which satisfies the balance constraints:

$$
\left(\boldsymbol{b}_{i}, L\right) \equiv 0 .
$$

The orthogonal complement of $L$ in the space with coordinates $\boldsymbol{\mu}=\boldsymbol{\nabla} G$ defines then the quasi-equilibrium manifold $\Omega_{L}$. For the actual computations, one requires the inversion from $\boldsymbol{\mu}$ to $\boldsymbol{c}$. Duality structure $\boldsymbol{\mu} \leftrightarrow \boldsymbol{c}$ is well studied by many authors [62,19]. 
Quasi-equilibrium projector. It is not sufficient to just derive the manifold, it is also required to define a projector which would transform the vector field defined on the space of concentrations to a vector field on the manifold. Quasi-equilibrium manifold consists of points which minimize $G$ on the affine spaces of the form $\boldsymbol{c}+L$. These affine planes are hypothetic planes of fast motions ( $G$ is decreasing in the course of the fast motions). Therefore, the quasi-equilibrium projector maps the whole space of concentrations on $\Omega_{L}$ parallel to $L$. The vector field is also projected onto the tangent space of $\Omega_{L}$ parallel to $L$.

Thus, the quasi-equilibrium approximation implies the decomposition of motions into the fast - parallel to $L$, and the slow - along the quasi-equilibrium manifold. In order to construct the quasi-equilibrium approximation, knowledge of reaction rate constants of "fast" reactions is not required (stoichiometric vectors of all these fast reaction are in $L$, $\gamma_{\text {fast }} \in L$, thus, knowledge of $L$ suffices), one only needs some confidence in that they all are sufficiently fast [74]. The quasi-equilibrium manifold itself is constructed based on the knowledge of $L$ and of $G$. Dynamics on the quasi-equilibrium manifold is defined as the quasi-equilibrium projection of the "slow component" of kinetic equations (4).

\subsection{Model equations}

The assumption behind the quasi-equilibrium is the hypothesis of the decomposition of motions into fast and slow. The quasi-equilibrium approximation itself describes slow motions. However, sometimes it becomes necessary to restore to the whole system, and to take into account the fast motions as well. With this, it is desirable to keep intact one of the important advantages of the quasi-equilibrium approximation - its independence of the rate constants of fast reactions. For this purpose, the detailed fast kinetics is replaced by a model equation (single relaxation time approximation).

Quasi-equilibrium models (QEM) are constructed as follows: For each concentration vector $\boldsymbol{c}$, consider the affine manifold, $\boldsymbol{c}+L$. Its intersection with the quasi-equilibrium manifold $\Omega_{L}$ consists of one point. This point delivers the minimum to $G$ on $\boldsymbol{c}+L$. Let us denote this point as $\boldsymbol{c}_{L}^{*}(\boldsymbol{c})$. The equation of the quasi-equilibrium model reads:

$$
\dot{\boldsymbol{c}}=-\frac{1}{\tau}\left[\boldsymbol{c}-\boldsymbol{c}_{L}^{*}(\boldsymbol{c})\right]+\sum_{\text {slow }} \gamma_{s} W_{s}\left(\boldsymbol{c}_{L}^{*}(\boldsymbol{c})\right)
$$

where $\tau>0$ is the relaxation time of the fast subsystem. Rates of slow reactions are computed in the points $\boldsymbol{c}_{L}^{*}(\boldsymbol{c})$ (the second term in the right hand side of Eq. (19), whereas the rapid motion is taken into account by a simple relaxational term (the first term in the right hand side of Eq. (19). The most famous model kinetic equation is the BGK equation in the theory of the Boltzmann equation [8]. The general theory of the quasi-equilibrium models, including proofs of their thermodynamic consistency, was constructed in $[27,29]$.

Single relaxation time gradient models (SRTGM) were considered in $[2,3,4]$ in the context of the lattice Boltzmann method for hydrodynamics. These models are aimed at improving 
the obvious drawback of quasi-equilibrium models (19): In order to construct the QEM, one needs to compute the function,

$$
\boldsymbol{c}_{L}^{*}(\boldsymbol{c})=\arg \min _{\boldsymbol{x} \in \boldsymbol{c}+L, \boldsymbol{x}>0} G(\boldsymbol{x})
$$

This is a convex programming problem. It does not always has a closed-form solution.

Let $\boldsymbol{g}_{1}, \ldots, \boldsymbol{g}_{k}$ is the orthonormal basis of $L$. We denote as $\boldsymbol{D}(\boldsymbol{c})$ the $k \times k$ matrix with the elements $\left(\boldsymbol{g}_{i}, \boldsymbol{H}_{\boldsymbol{c}} \boldsymbol{g}_{j}\right)$, where $\boldsymbol{H}_{\boldsymbol{c}}$ is the matrix of second derivatives of $G(7)$. Let $\boldsymbol{C}(\boldsymbol{c})$ be the inverse of $\boldsymbol{D}(\boldsymbol{c})$. The single relaxation time gradient model has the form:

$$
\dot{\boldsymbol{c}}=-\frac{1}{\tau} \sum_{i, j} \boldsymbol{g}_{i} \boldsymbol{C}(\boldsymbol{c})_{i j}\left(\boldsymbol{g}_{j}, \nabla G\right)+\sum_{\text {slow }} \boldsymbol{\gamma}_{s} W_{s}(\boldsymbol{c}) .
$$

The first term drives the system to the minimum of $G$ on $c+L$, it does not require solving the problem (20), and its spectrum in the quasi-equilibrium is the same as in the quasi-equilibrium model (19). Note that the slow component is evaluated in the "current" state $\boldsymbol{c}$.

The models (19) and (21) lift the quasi-equilibrium approximation to a kinetic equation by approximating the fast dynamics with a single "reaction rate constant" - relaxation time $\tau$.

\subsection{Quasi-steady state approximation}

The quasi-steady state approximation (QSS) is a tool used in a huge amount of works. Let us split the list of species in two groups: The basic and the intermediate (radicals etc). Concentration vectors are denoted accordingly, $\boldsymbol{c}^{\mathrm{s}}$ (slow, basic species), and $\boldsymbol{c}^{\mathrm{f}}$ (fast, intermediate species). The concentration vector $\boldsymbol{c}$ is the direct sum, $\boldsymbol{c}=\boldsymbol{c}^{\mathrm{s}} \oplus \boldsymbol{c}^{\mathrm{f}}$. The fast subsystem is Eq. (4) for the component $\boldsymbol{c}^{\mathrm{f}}$ at fixed values of $\boldsymbol{c}^{\mathrm{s}}$. If it happens that this way defined fast subsystem relaxes to a stationary state, $\boldsymbol{c}^{\mathrm{f}} \rightarrow \boldsymbol{c}_{\mathrm{qss}}^{\mathrm{f}}\left(\boldsymbol{c}^{\mathrm{s}}\right)$, then the assumption that $\boldsymbol{c}^{\mathrm{f}}=\boldsymbol{c}_{\mathrm{qss}}^{\mathrm{f}}(\boldsymbol{c})$ is precisely the QSS assumption. The slow subsystem is the part of the system (4) for $\boldsymbol{c}^{\mathrm{s}}$, in the right hand side of which the component $\boldsymbol{c}^{\mathrm{f}}$ is replaced with $\boldsymbol{c}_{\mathrm{qss}}^{\mathrm{f}}(\boldsymbol{c})$. Thus, $\boldsymbol{J}=\boldsymbol{J}_{\mathrm{s}} \oplus \boldsymbol{J}_{\mathrm{f}}$, where

$$
\begin{aligned}
& \dot{\boldsymbol{c}}^{\mathrm{f}}=\boldsymbol{J}_{\mathrm{f}}\left(\boldsymbol{c}^{\mathrm{s}} \oplus \boldsymbol{c}^{\mathrm{f}}\right), \boldsymbol{c}^{\mathrm{s}}=\mathrm{const} ; \quad \boldsymbol{c}^{\mathrm{f}} \rightarrow \boldsymbol{c}_{\mathrm{qss}}^{\mathrm{f}}\left(\boldsymbol{c}^{\mathrm{s}}\right) ; \\
& \dot{\boldsymbol{c}}^{\mathrm{s}}=\boldsymbol{J}_{\mathrm{s}}\left(\boldsymbol{c}^{\mathrm{s}} \oplus \boldsymbol{c}_{\mathrm{qss}}^{\mathrm{f}}\left(\boldsymbol{c}^{\mathrm{s}}\right)\right) .
\end{aligned}
$$

Bifurcations in the system (22) under variation of $\boldsymbol{c}^{\mathrm{s}}$ as a parameter are confronted to kinetic critical phenomena. Studies of more complicated dynamic phenomena in the fast subsystem (22) require various techniques of averaging, stability analysis of the averaged quantities etc. 
Various versions of the QSS method are well possible, and are actually used widely, for example, the hierarchical QSS method. There, one defines not a single fast subsystem but a hierarchy of them, $\boldsymbol{c}^{\mathrm{f}_{1}}, \ldots, \boldsymbol{c}^{\mathrm{f}_{k}}$. Each subsystem $\boldsymbol{c}^{\mathrm{f}_{i}}$ is regarded as a slow system for all the foregoing subsystems, and it is regarded as a fast subsystem for the following members of the hierarchy. Instead of one system of equations (22), a hierarchy of systems of lower-dimensional equations is considered, each of these subsystem is easier to study analytically.

Theory of singularly perturbed systems of ordinary differential equations is used to provide a mathematical background and further development of the QSS approximation [10,68]. In spite of a broad literature on this subject, it remains, in general, unclear, what is the smallness parameter that separates the intermediate (fast) species from the basic (slow). Reaction rate constants cannot be such a parameter (unlike in the case of the quasiequilibrium). Indeed, intermediate species participate in the same reactions, as the basic species (for example, $\mathrm{H}_{2} \rightleftharpoons 2 \mathrm{H}, \mathrm{H}+\mathrm{O}_{2} \rightleftharpoons \mathrm{OH}+\mathrm{O}$ ). It is therefore incorrect to state that $\boldsymbol{c}^{\mathrm{f}}$ evolve faster than $\boldsymbol{c}^{\mathrm{s}}$. In the sense of reaction rate constants, $\boldsymbol{c}^{\mathrm{f}}$ is not faster.

For catalytic reactions, it is not difficult to figure out what is the smallness parameter that separates the intermediate species from the basic, and which allows to upgrade the QSS assumption to a singular perturbation theory rigorously [77]. This smallness parameter is the ratio of balances: Intermediate species include the catalyst, and their total amount is simply significantly less than the amount of all the $\boldsymbol{c}_{i}$ 's. After renormalizing to the variables of one order of magnitude, the small parameter appears explicitly.

For usual radicals, the origin of the smallness parameter is quite similar. There are much less radicals than the basic species (otherwise, the QSS assumption is inapplicable). In the case of radicals, however, the smallness parameter cannot be extracted directly from balances $B_{i}(2)$. Instead, one can come up with a thermodynamic estimate: Function $G$ decreases in the course of reactions, whereupon we obtain the limiting estimate of concentrations of any specie:

$$
c_{i} \leq \max _{G(\boldsymbol{c}) \leq G(\boldsymbol{c}(0))} c_{i},
$$

where $\boldsymbol{c}(0)$ is the initial composition. If the concentration $c_{\mathrm{R}}$ of the radical $\mathrm{R}$ is small both initially and in the equilibrium, then it should remain small also along the path to the equilibrium. For example, in the case of ideal $G(9)$ under relevant conditions, for any $t>0$, the following inequality is valid:

$$
c_{\mathrm{R}}\left[\ln \left(c_{\mathrm{R}}(t) / c_{\mathrm{R}}^{\mathrm{eq}}\right)-1\right] \leq G(\boldsymbol{c}(0)) .
$$

Inequality (25) provides the simplest (but rather coarse) thermodynamic estimate of $c_{\mathrm{R}}(t)$ in terms of $G(\boldsymbol{c}(0))$ and $c_{\mathrm{R}}^{\text {eq }}$ uniformly for $t>0$. Complete theory of thermodynamic estimates of dynamics has been developed in [23]. One can also do computations without a priori estimations, if one accepts the QSS assumption until the values $\boldsymbol{c}^{\mathrm{f}}$ stay sufficiently small. 
Let us assume that an a priori estimate has been found, $c_{i}(t) \leq c_{i} \max$, for each $c_{i}$. These estimate may depend on the initial conditions, thermodynamic data etc. With these estimates, we are able to renormalize the variables in the kinetic equations (4) in such a way that renormalized variables take their values from the unit segment $[0,1]$ : $\tilde{c}_{i}=c_{i} / c_{i \max }$. Then the system (4) can be written as follows:

$$
\frac{d \tilde{c}_{i}}{d t}=\frac{1}{c_{i \max }} J_{i}(\boldsymbol{c})
$$

The system of dimensionless parameters, $\epsilon_{i}=c_{i \max } / \max _{i} c_{i \text { max }}$ defines a hierarchy of relaxation times, and with its help one can establish various realizations of the QSS approximation. The simplest version is the standard QSS assumption: Parameters $\epsilon_{i}$ are separated in two groups, the smaller ones, and of the order 1. Accordingly, the concentration vector is split into $\boldsymbol{c}^{\mathrm{s}} \oplus \boldsymbol{c}^{\mathrm{f}}$. Various hierarchical QSS are possible, with this, the problem becomes more tractable analytically.

Corrections to the QSS approximation can be addressed in various ways (see, e. g., [72,70]). There exist a variety of ways to introduce the smallness parameter into kinetic equations, and one can find applications to each of the realizations. However, the two particular realizations remain basic for chemical kinetics: (i) Fast reactions (under a given thermodynamic data); (ii) Small concentrations. In the first case, one is led to the quasiequilibrium approximation, in the second case - to the classical QSS assumption. Both of these approximations allow for hierarchical realizations, those which include not just two but many relaxation time scales. Such a multi-scale approach essentially simplifies analytical studies of the problem.

The method of invariant manifold which we present below in the section 6 allows to use both the QE and the QSS as initial approximations in the iterational process of seeking slow invariant manifolds. It is also possible to use a different initial ansatz chosen by a physical intuition, like, for example, the Tamm-Mott-Smith approximation in the theory of strong shock waves [24].

\subsection{Methods based on spectral decomposition of Jacobian fields}

The idea to use the spectral decomposition of Jacobian fields in the problem of separating the motions into fast and slow originates from methods of analysis of stiff systems [22], and from methods of sensitivity analysis in control theory [64]. There are two basic statements of the problem for these methods: (i) The problem of the slow manifold, and (ii) The problem of a complete decomposition (complete integrability) of kinetic equations. The first of these problems consists in constructing the slow manifold $\Omega$, and a decomposition of motions into the fast one - towards $\Omega$, and the slow one - along $\boldsymbol{\Omega}$ [61]. The second of these problems consists in a transformation of kinetic equations (4) to a diagonal form, $\dot{\zeta}_{i}=f_{i}\left(\zeta_{i}\right)$ (so-called full nonlinear lumping or modes decoupling, [56,59,71]). Clearly, if one finds a sufficiently explicit solution to the second problem, then the system (4) is completely integrable, and nothing more is needed, the result has to be simply used. The 
question is only to what extend such a solution can be possible, and how difficult it would be as compared to the first problem to find it.

One of the currently most popular methods is the construction of the so-called intrinsic low-dimensional manifold (ILDM, [61]). This method is based on the following geometric picture: For each point $\boldsymbol{c}$, one defines the Jacobian matrix of Eq. (4), $\boldsymbol{F} \boldsymbol{c} \equiv \partial \boldsymbol{J}(\boldsymbol{c}) / \partial \boldsymbol{c}$. One assumes that, in the domain of interest, the eigenvalues of $\boldsymbol{F}_{\boldsymbol{c}}$ are separated into two groups, $\lambda_{i}^{\mathrm{s}}$ and $\lambda_{j}^{\mathrm{f}}$, and that the following inequalities are valid:

$$
\operatorname{Re} \lambda_{i}^{\mathrm{s}} \geq a>b \geq \operatorname{Re} \lambda_{j}^{\mathrm{f}}, a \gg b, b<0 .
$$

Let us denote as $L_{\boldsymbol{c}}^{\mathrm{s}}$ and $L_{\boldsymbol{c}}^{\mathrm{f}}$ the invariant subspaces corresponding to $\lambda^{\mathrm{s}}$ and $\lambda^{\mathrm{f}}$, respectively, and let $\boldsymbol{Z}_{\boldsymbol{c}}^{\mathrm{s}}$ and $\boldsymbol{Z}_{\boldsymbol{c}}^{\mathrm{f}}$ be the corresponding spectral projectors, $\boldsymbol{Z}_{\boldsymbol{c}}^{\mathrm{s}} L_{\boldsymbol{c}}^{\mathrm{s}}=L_{\boldsymbol{c}}^{\mathrm{s}}$, $\boldsymbol{Z}_{\boldsymbol{c}}^{\mathrm{f}} L_{\boldsymbol{c}}^{\mathrm{f}}=L_{\boldsymbol{c}}^{\mathrm{f}}, \boldsymbol{Z}_{\boldsymbol{c}}^{\mathrm{s}} L_{\boldsymbol{c}}^{\mathrm{f}}=\boldsymbol{Z}_{\boldsymbol{c}}^{\mathrm{f}} L_{\boldsymbol{c}}^{\mathrm{s}}=\{0\}, \boldsymbol{Z}_{\boldsymbol{c}}^{\mathrm{s}}+\boldsymbol{Z}_{\boldsymbol{c}}^{\mathrm{f}}=1$. Operator $\boldsymbol{Z}_{\boldsymbol{c}}^{\mathrm{s}}$ projects onto the subspace of "slow modes" $L_{\boldsymbol{c}}^{\mathrm{s}}$, and it annihilates the "fast modes" $L_{\boldsymbol{c}}^{\mathrm{f}}$. Operator $\boldsymbol{Z}_{\boldsymbol{c}}^{\mathrm{f}}$ does the opposite, it projects onto fast modes, and it annihilates the slow modes. The basic equation of the ILDM reads:

$$
Z_{\boldsymbol{c}}^{\mathrm{f}} \boldsymbol{J}(\boldsymbol{c})=0
$$

In this equation, the unknown is the concentration vector $\boldsymbol{c}$. The set of solutions to Eq. (27) is the ILDM manifold $\boldsymbol{\Omega}_{\mathrm{ildm}}$.

For linear systems, $\boldsymbol{F}_{\boldsymbol{c}}, \boldsymbol{Z}_{\boldsymbol{c}}^{\mathrm{s}}$, and $\boldsymbol{Z}_{\boldsymbol{c}}^{\mathrm{f}}$, do not depend on $\boldsymbol{c}$, and $\boldsymbol{\Omega}_{\mathrm{ildm}}=\boldsymbol{c}^{\mathrm{eq}}+L^{\mathrm{s}}$. On the other hand, obviously, $\boldsymbol{c}^{\text {eq }} \in \Omega_{\text {ildm }}$. Therefore, procedures of solving of Eq. (27) can be initiated by choosing the linear approximation, $\Omega_{\mathrm{ildm}}^{(0)}=\boldsymbol{c}^{\mathrm{eq}}+L_{\boldsymbol{c}^{\mathrm{eq}}}^{\mathrm{s}}$, in the neighborhood of the equilibrium $\boldsymbol{c}^{\mathrm{eq}}$, and then continued parametrically into the nonlinear domain. Computational technologies of a continuation of solutions with respect to parameters are well developed (see, for example, $[55,65]$ ). The problem of the relevant parameterization is solved locally: In the neighborhood of a given point $\boldsymbol{c}^{0}$ one can choose $\boldsymbol{Z}_{\boldsymbol{c}}^{\mathrm{s}}\left(\boldsymbol{c}-\boldsymbol{c}^{0}\right)$ for a characterization of the vector $\boldsymbol{c}$. In this case, the space of parameters is $L_{\boldsymbol{c}}^{\mathrm{s}}$. There exist other, physically motivated ways to parameterize manifolds ([24]; see also section 4.1 below).

There are two drawbacks of the ILDM method which call for its refinement: (i) "Intrinsic" does not imply "invariant". Eq. (27) is not invariant of the dynamics (4). If one differentiates Eq. (27) in time due to Eq. (4), one obtains a new equation which is the implication of Eq. (27) only for linear systems. In a general case, the motion $\boldsymbol{c}(t)$ takes off the $\boldsymbol{\Omega}_{\text {ildm }}$. Invariance of a manifold $\boldsymbol{\Omega}$ means that $\boldsymbol{J}(\boldsymbol{c})$ touches $\boldsymbol{\Omega}$ in every point $\boldsymbol{c} \in \boldsymbol{\Omega}$. It remains unclear how the ILDM (27) corresponds with this condition. Thus, from the dynamical perspective, the status of the ILDM remains not well defined, or "ILDM is ILDM", defined self-consistently by Eq. (27), and that is all what can be said about it. (ii) From the geometrical standpoint, spectral decomposition of Jacobian fields is not the most attractive way to compute manifolds. If we are interested in the behavior of trajectories, how they converge or diverge, then one should consider the symmetrized part of $\boldsymbol{F}_{\boldsymbol{c}}$, rather than $\boldsymbol{F}_{\boldsymbol{c}}$ itself. 
Symmetric part, $\boldsymbol{F}_{\boldsymbol{C}}^{\text {sym }}=(1 / 2)\left(\boldsymbol{F}_{\boldsymbol{c}}^{\dagger}+\boldsymbol{F}_{\boldsymbol{c}}\right)$, defines the dynamics of the distance between two solutions, $\boldsymbol{c}$ and $\boldsymbol{c}^{\prime}$, in a given local Euclidean metrics. Skew-symmetric part defines rotations. If we want to study manifolds based on the argument about convergence/divergence of trajectories, then we should use in Eq. (27) the spectral projector $\boldsymbol{Z}_{\boldsymbol{c}}^{\text {fsym }}$ for the operator $\boldsymbol{F}_{\boldsymbol{c}}^{\text {sym }}$. This, by the way, is also a significant simplification from the standpoint of computations. It remains to choose the metrics. This choice is unambiguous from the thermodynamic perspective. In fact, there is only one choice which fits into the physical meaning of the problem, this is the metrics associated with the thermodynamic (or entropic) scalar product,

$$
\langle\boldsymbol{x}, \boldsymbol{y}\rangle=\left(\boldsymbol{x}, \boldsymbol{H}_{\boldsymbol{c}} \boldsymbol{y}\right)
$$

where $\boldsymbol{H}_{\boldsymbol{c}}$ is the matrix of second-order derivatives of $G(7)$. In the equilibrium, operator $\boldsymbol{F}_{\boldsymbol{c}^{\text {eq }}}$ is selfadjoint with respect to this scalar product (Onsager's reciprocity relations). Therefore, the behavior of the ILDM in the vicinity of the equilibrium does not alter under the replacement, $\boldsymbol{F}_{\boldsymbol{c}^{\mathrm{eq}}}=\boldsymbol{F}_{\boldsymbol{C}^{\mathrm{eq}}}^{\text {sym }}$. In terms of usual matrix representation, we have:

$$
\boldsymbol{F}_{\boldsymbol{c}}^{\mathrm{sym}}=\frac{1}{2}\left(\boldsymbol{F}_{\boldsymbol{c}}+\boldsymbol{H}_{\boldsymbol{c}}^{-1} \boldsymbol{F}_{\boldsymbol{c}}^{T} \boldsymbol{H}_{\boldsymbol{c}}\right)
$$

where $\boldsymbol{F}_{\boldsymbol{c}}^{T}$ is the ordinary transposition.

The ILDM constructed with the help of the symmetrized Jacobian field will be termed the symmetric entropic intrinsic low-dimensional manifold (SEILDM). Selfadjointness of $\boldsymbol{F}_{\boldsymbol{C}}^{\text {sym }}(29)$ with respect to the thermodynamic scalar product (28) simplifies considerably computations of spectral decomposition. Moreover, it becomes necessary to do spectral decomposition in only one point - in the equilibrium. Perturbation theory for selfadjoint operators is a very well developed subject [53], which makes it possible to easily extend the spectral decomposition with respect to parameters. A more detailed discussion of the selfadjoint linearization will be given below in section 5.2.

Thus, when the geometric picture behind the decomposition of motions is specified, the physical significance of the ILDM becomes more transparent, and it leads to its modification into the SEILDM. This also gains simplicity in the implementation by switching from non-selfadjoint spectral problems to selfadjoint. The quantitative estimate of this simplification is readily available: Let $d$ be the dimension of the phase space, and $k$ the dimension of the ILDM $\left(k=\operatorname{dim} L_{\boldsymbol{c}}^{\mathrm{s}}\right)$. The space of all the projectors $\boldsymbol{Z}$ with the $k$ dimensional image has the dimension $D=2 k(d-k)$. The space of all the selfadjoint projectors with the $k$-dimensional image has the dimension $D^{\text {sym }}=k(d-k)$. For $d=20$ and $k=3$, we have $D=102$ and $D^{\text {sym }}=51$. When the spectral decomposition by means of parametric extension is addressed, one considers equations of the form:

$$
\frac{d \boldsymbol{Z}_{\boldsymbol{c}(\tau)}^{\mathrm{s}}}{d \tau}=\boldsymbol{\Psi}^{\mathrm{s}}\left(\frac{d \boldsymbol{c}}{d \tau}, \boldsymbol{Z}_{\boldsymbol{c}(\tau)}^{\mathrm{s}}, \boldsymbol{F}_{\boldsymbol{c}(\tau)}, \nabla \boldsymbol{F}_{\boldsymbol{c}(\tau)}\right)
$$

where $\tau$ is the parameter, and $\boldsymbol{\nabla} \boldsymbol{F}_{\boldsymbol{c}}=\boldsymbol{\nabla} \boldsymbol{\nabla} \boldsymbol{J}(\boldsymbol{c})$ is the differential of the Jacobian field. 
For the selfadjoint case, where we use $=\boldsymbol{F}_{\boldsymbol{c}}^{\text {sym }}$ instead of $\boldsymbol{F} \boldsymbol{c}$, this system of equations has twice less independent variables, and also the right hand is of a simpler structure.

It is more difficult to improve on the first of the remarks (ILDM is not invariant). The following naive approach may seem possible:

(i) Take $\Omega_{\text {ildm }}=\boldsymbol{c}^{\mathrm{eq}}+L_{\boldsymbol{c}^{\mathrm{eq}}}^{\mathrm{s}}$ in a neighborhood $U$ of the equilibrium $\boldsymbol{c}^{\mathrm{eq}}$. [This is also a useful initial approximation for solving Eq. (27)].

(ii) Instead of computing the solution to Eq. (27), integrate the kinetic equations (4) backwards in the time. It is sufficient to take initial conditions $\boldsymbol{c}(0)$ from a dense set on the boundary, $\partial U \cap\left(\boldsymbol{c}^{\mathrm{eq}}+L_{\boldsymbol{c}^{\mathrm{eq}}}^{\mathrm{s}}\right)$, and to compute solutions $\boldsymbol{c}(t), t<0$.

(iii) Consider the obtained set of trajectories as an approximation of the slow invariant manifold.

This approach will guarantee invariance, by construction, but it is prone to pitfalls in what concerns the slowness. Indeed, the integration backwards in the time will see exponentially divergent trajectories, if they were exponentially converging in the normal time progress. This way one finds some invariant manifold which touches $\boldsymbol{c}^{\mathrm{eq}}+L_{\boldsymbol{c}^{\mathrm{eq}}}^{\mathrm{s}}$ in the equilibrium. Unfortunately, there are infinitely many such manifolds, and they fill out almost all the space of concentrations. However, we must select the slow component of motions. Such a regularization is possible. Indeed, let us replace in Eq. (4) the vector field $\boldsymbol{J}(\boldsymbol{c})$ by the vector field $\boldsymbol{Z}_{\boldsymbol{c}}^{\text {ssym }} \boldsymbol{J}(\boldsymbol{c})$, and obtain a regularized kinetic equation,

$$
\dot{c}=Z_{\boldsymbol{c}}^{\mathrm{ssym}} \boldsymbol{J}(\boldsymbol{c}) .
$$

Let us replace integration backwards in time of the kinetic equation (4) in the naive approach described above by integration backwards in time of the regularized kinetic equation (31). With this, we obtain a rather convincing version of the ILDM (SEILDM). Using Eq. (30), one also can write down an equation for the projector $\boldsymbol{Z}_{\boldsymbol{c}}^{\text {ssym }}$, putting $\tau=t$. Replacement of Eq. (4) by Eq. (31) also makes the integration backwards in time in the naive approach more stable. However, regularization will again conflict with invariance. The "naive refinement" after the regularization (31) produces just a slightly different version of the ILDM (or SEILDM) but it does not construct the slow invariant manifold. So, where is the way out? We believe that the ILDM and its version SEILDM are, in general, good initial approximations of the slow manifold. However, if one is indeed interested in finding the invariant manifold, one has to write out the true condition of invariance and solve it. As for the initial approximation for the method of invariant manifold one can use any ansatz, in particular, the SEILDM.

The problem of a complete decomposition of kinetic equations can be solved indeed in some cases. The first such solution was the spectral decomposition for linear systems [75]. Decomposition is sometimes possible also for nonlinear systems ([59]; [71]). The most famous example of a complete decomposition of infinite-dimensional kinetic equation is the complete integrability of the space-independent Boltzmann equation for Maxwell's molecules found in [9]. However, in a general case, there exist no analytical, not even a twice differen- 
tiable transformation which would decouple modes. The well known Grobman-Hartman theorem $[43,44]$ states only the existence of a continuous transform which decomposes modes in a neighborhood of the equilibrium. For example, the analytic planar system, $d x / d t=-x, d y / d t=-2 y+x^{2}$, is not $C^{2}$ linearizable. These problems remain of interest [15]. Therefore, in particular, it becomes quite ineffective to construct such a transformation in a form of a series. It is more effective to solve a simpler problem of extraction of a slow invariant manifold [7].

Sensitivity analysis $[64,63,56]$ makes it possible to select essential variables and reactions, and to decompose motions into fast and slow. In a sense, the ILDM method is a development of the sensitivity analysis. In particular, the computational singular perturbation (CSP) method of [56] includes ILDM (or any other reasonable initial choice of the manifold) into a procedure of consequent refinements. Recently, a further step in this direction was done in [78]. In this work, the authors use a nonlocal in time criterion of closeness of solutions of the full and of the reduced systems of chemical kinetics. They require not just a closeness of derivatives but a true closeness of the dynamics.

Let us be interested in the dynamics of the concentrations of just a few species, $A_{1}, \ldots, A_{p}$, whereas the rest of the species, $\mathrm{A}_{p+1}, \ldots, \mathrm{A}_{n}$ are used for building the kinetic equation, and for understanding the process. Let $\boldsymbol{c}_{\text {goal }}$ be the concentration vector with components $c_{1}, \ldots, c_{p}, \boldsymbol{c}_{\text {goal }}(t)$ be the corresponding components of the solution to Eq. (4), and $\boldsymbol{c}_{\text {goal }}^{\mathrm{red}}$ be the solution to the simplified model with corresponding initial conditions. [78] suggest to minimize the difference between $\boldsymbol{c}_{\text {goal }}(t)$ and $\boldsymbol{c}_{\text {goal }}^{\text {red }}$ on the segment $t \in[0, T]: \| \boldsymbol{c}_{\text {goal }}(t)-$ $c_{\text {goal }}^{\text {red }} \| \rightarrow$ min. In the course of the optimization under certain restrictions one selects the optimal (or appropriate) reduced model. The sequential quadratic programming method and heuristic rules of sorting the reactions, substances etc were used. In the result, for some stiff systems studied, one avoids typical pitfalls of the local sensitivity analysis. In simpler situations this method should give similar results as the local methods.

\subsection{Thermodynamic criteria for selection of important reactions}

One of the problems addressed by the sensitivity analysis is the selection of the important and discarding the unimportant reactions. [12] suggested a simple principle to compare importance of different reactions according to their contribution to the entropy production (or, which is the same, according to their contribution to $\dot{G}$ ). Based on this principle, [17] described domains of parameters in which the reaction of hydrogen oxidation, $\mathrm{H}_{2}+\mathrm{O}_{2}+\mathrm{M}$, proceeds due to different mechanisms. For each elementary reaction, he has derived the domain inside which the contribution of this reaction is essential (nonnegligible). Due to its simplicity, this entropy production principle is especially well suited for analysis of complex problems. In particular, recently, a version of the entropy production principle was used in the problem of selection of boundary conditions for Grad's moment equations $[69,42]$. For ideal systems $(9)$, the contribution of the $s$ th reaction to $\dot{G}$ has a particularly 
simple form:

$$
\dot{G}_{s}=-W_{s} \ln \left(\frac{W_{s}^{+}}{W_{s}^{-}}\right), \dot{G}=\sum_{s=1}^{r} \dot{G}_{s}
$$

For nonideal systems, the corresponding expressions (13) are also not too complicated.

\section{Outline of the method of invariant manifold}

In many cases, dynamics of the $d$-dimensional system (4) leads to a manifold of a lower dimension. Intuitively, a typical phase trajectory behaves as follows: Given the initial state $\boldsymbol{c}(0)$ at $t=0$, and after some period of time, the trajectory comes close to some low-dimensional manifold $\Omega$, and after that proceeds towards the equilibrium essentially along this manifold. The goal is to construct this manifold.

The starting point of our approach is based on a formulation of the two main requirements:

(i). Dynamic invariance: The manifold $\Omega$ should be (positively) invariant under the dynamics of the originating system (4): If $\boldsymbol{c}(0) \in \boldsymbol{\Omega}$, then $\boldsymbol{c}(t) \in \boldsymbol{\Omega}$ for each $t>0$.

(ii). Thermodynamic consistency of the reduced dynamics: Let some (not obligatory invariant) manifold $\Omega$ is considered as a manifold of reduced description. We should define a set of linear operators, $\boldsymbol{P} \boldsymbol{c}$, labeled by the states $\boldsymbol{c} \in \boldsymbol{\Omega}$, which project the vectors $\boldsymbol{J}(\boldsymbol{c})$, $c \in \Omega$ onto the tangent bundle of the manifold $\boldsymbol{\Omega}$, thereby generating the induced vector field, $\boldsymbol{P}_{\boldsymbol{c}} \boldsymbol{J}(\boldsymbol{c}), \boldsymbol{c} \in \boldsymbol{\Omega}$. This induced vector field on the tangent bundle of the manifold $\boldsymbol{\Omega}$ is identified with the reduced dynamics along the manifold $\boldsymbol{\Omega}$. The thermodynamicity requirement for this induced vector field reads

$$
\left(\boldsymbol{\nabla} G(\boldsymbol{c}), \boldsymbol{P}_{\boldsymbol{c}} \boldsymbol{J}(\boldsymbol{c})\right) \leq 0, \text { for each } \boldsymbol{c} \in \boldsymbol{\Omega}
$$

In order to meet these requirements, the method of invariant manifold suggests two complementary procedures:

(i). To treat the condition of dynamic invariance as an equation, and to solve it iteratively by a Newton method. This procedure is geometric in its nature, and it does not use the time dependence and small parameters.

(ii). Given an approximate manifold of reduced description, to construct the projector satisfying the condition (33) in a way which does not depend on the vector field $\boldsymbol{J}$.

We shall now outline both these procedures starting with the second. The solution consists, in the first place, in formulating the thermodynamic condition which should be met by the projectors $\boldsymbol{P}_{\boldsymbol{c}}$ : For each $\boldsymbol{c} \in \boldsymbol{\Omega}$, let us consider the linear functional

$$
M_{\boldsymbol{c}}^{*}(\boldsymbol{x})=(\boldsymbol{\nabla} G(\boldsymbol{c}), \boldsymbol{x})
$$


Then the thermodynamic condition for the projectors reads:

$$
\operatorname{ker} \boldsymbol{P} c \subseteq \operatorname{ker} M_{\boldsymbol{c}}^{*}, \text { for each } \boldsymbol{c} \in \boldsymbol{\Omega}
$$

Here $\operatorname{ker} \boldsymbol{P} \boldsymbol{c}$ is the null space of the projector, and $\operatorname{ker} M_{\boldsymbol{c}}^{*}$ is the hyperplane orthogonal to the vector $M_{\boldsymbol{c}}^{*}$. It has been shown [24,28] that the condition (35) is the necessary and sufficient condition to establish the thermodynamic induce vector field on the given manifold $\boldsymbol{\Omega}$ for all possible dissipative vector fields $\boldsymbol{J}$ simultaneously.

Let us now turn to the requirement of invariance. By a definition, the manifold $\Omega$ is invariant with respect to the vector field $\boldsymbol{J}$ if and only if the following equality is true:

$$
[1-\boldsymbol{P}] \boldsymbol{J}(\boldsymbol{c})=0, \text { for each } \boldsymbol{c} \in \boldsymbol{\Omega} \text {. }
$$

In this expression $\boldsymbol{P}$ is an arbitrary projector on the tangent bundle of the manifold $\boldsymbol{\Omega}$. It has been suggested to consider the condition (36) as an equation to be solved iteratively starting with some appropriate initial manifold.

Iterations for the invariance equation (36) are considered in the section 5 . The next section presents construction of the thermodynamic projector using a specific parameterization of manifolds.

\section{Thermodynamic projector}

\subsection{Thermodynamic parameterization}

In this section, $\boldsymbol{\Omega}$ denotes a generic $p$-dimensional manifold. First, it should be mentioned that any parameterization of $\Omega$ generates a certain projector, and thereby a certain reduced dynamics. Indeed, let us consider a set of $m$ independent functionals $M(\boldsymbol{c})=\left\{M_{1}(\boldsymbol{c}), \ldots, M_{p}(\boldsymbol{c})\right\}$, and let us assume that they form a coordinate system on $\boldsymbol{\Omega}$ in such a way that $\boldsymbol{\Omega}=\boldsymbol{c}(M)$, where $\boldsymbol{c}(M)$ is a vector function of the parameters $M_{1}, \ldots, M_{p}$. Then the projector associated with this parameterization reads:

$$
\boldsymbol{P}_{\boldsymbol{c}(M)} \boldsymbol{x}=\sum_{i=1}^{p} \frac{\partial \boldsymbol{c}(M)}{\partial M_{i}}\left(\left.\boldsymbol{\nabla} M_{i}\right|_{\boldsymbol{c}(M)}, \boldsymbol{x}\right),
$$

where $N_{i j}^{-1}$ is the inverse to the $p \times p$ matrix:

$$
\boldsymbol{N}(M)=\left\|\left(\boldsymbol{\nabla} M_{i}, \partial \boldsymbol{c} / \partial M_{j}\right)\right\|
$$

This somewhat involved notation is intended to stress that the projector (37) is dictated by

the choice of the parameterization. Subsequently, the induced vector field of the reduced 
dynamics is found by applying projectors (37) on the vectors $\boldsymbol{J}(\boldsymbol{c}(M))$, thereby inducing the reduced dynamics in terms of the parameters $M$ as follows:

$$
\dot{M}_{i}=\sum_{j=1}^{p} N_{i j}^{-1}(M)\left(\left.\nabla M_{j}\right|_{\boldsymbol{c}(M)}, \boldsymbol{J}(\boldsymbol{c}(M))\right)
$$

Depending on the choice of the parameterization, dynamic equations (39) are (or are not) consistent with the thermodynamic requirement (33). The thermodynamic parameterization makes use of the condition (35) in order to establish the thermodynamic projector. Specializing to the case (37), let us consider the linear functionals,

$$
\left.D M_{i}\right|_{\boldsymbol{c}(M)}(\boldsymbol{x})=\left(\left.\boldsymbol{\nabla} M_{i}\right|_{\boldsymbol{c}(M)}, \boldsymbol{x}\right)
$$

Then the condition (35) takes the form:

$$
\bigcap_{i=1}^{p} \operatorname{ker} D M_{i} \mid \boldsymbol{c}(M) \subseteq \operatorname{ker} M_{\boldsymbol{c}(M)}^{*},
$$

that is, the intersection of null spaces of the functionals (40) should belong to the null space of the differential of the Lyapunov function $G$, in each point of the manifold $\Omega$.

In practice, in order to construct the thermodynamic parameterization, we take the following set of functionals in each point $\boldsymbol{c}$ of the manifold $\Omega$ :

$$
\begin{aligned}
M_{1}(\boldsymbol{x}) & =M_{\boldsymbol{c}}^{*}(\boldsymbol{x}), \boldsymbol{c} \in \boldsymbol{\Omega} \\
M_{i}(\boldsymbol{x}) & =\left(\boldsymbol{m}_{i}, \boldsymbol{x}\right), i=2, \ldots, p
\end{aligned}
$$

It is required that vectors $\boldsymbol{\nabla} G(\boldsymbol{c}), \boldsymbol{m}_{2}, \ldots, \boldsymbol{m}_{p}$ are linearly independent in each state $\boldsymbol{c} \in \boldsymbol{\Omega}$. Inclusion of the functionals (34) as a part of the system (42) and (43) implies the thermodynamic condition (41). Also, any linear combination of the parameter set (42), (43) will meet the thermodynamicity requirement.

It is important to notice here that the thermodynamic condition is satisfied whatsoever the functionals $M_{2}, \ldots, M_{p}$ are. This is very convenient for it gives an opportunity to take into account the conserved quantities correctly. The manifolds we are going to deal with should be consistent with the conservation laws (2). While the explicit characterization of the phase space $\boldsymbol{V}$ is a problem on its own, in practice, it is customary to work in the $n$-dimensional space while keeping the constraints (2) explicitly on each step of the construction. For this technical reason, it is convenient to consider manifolds of the dimension $p>l$, where $l$ is the number of conservation laws, in the $n$-dimensional space rather than in the phase space $\boldsymbol{V}$. The thermodynamic parameterization is then concordant also with the conservation laws if $l$ of the linear functionals (43) are identified with the conservation laws. In the sequel, only projectors consistent with conservation laws are considered. 
Very frequently, the manifold $\boldsymbol{\Omega}$ is represented as a $p$-parametric family $\boldsymbol{c}\left(a_{1}, \ldots, a_{p}\right)$, where $a_{i}$ are coordinates on the manifold. The thermodynamic re-parameterization suggests a representation of the coordinates $a_{i}$ in terms of $M_{\boldsymbol{c}}^{*}, M_{2}, \ldots, M_{p}$ (42), (43). While the explicit construction of these functions may be a formidable task, we notice that the construction of the thermodynamic projector of the form (37) and of the dynamic equations (39) is relatively easy because only the derivatives $\partial \boldsymbol{c} / \partial M_{i}$ enter these expressions. This point was discussed in a detail in [24,28].

\subsection{Decomposition of motions: Thermodynamics}

Finally, let us discuss how the thermodynamic projector is related to the decomposition of motions. Assuming that the decomposition of motions near the manifold $\Omega$ is true indeed, let us consider states which were initially close enough to the manifold $\boldsymbol{\Omega}$. Even without knowing the details about the evolution of the states towards $\Omega$, we know that the Lyapunov function $G$ was decreasing in the course of this evolution. Let us consider a set of states $\boldsymbol{U}_{\boldsymbol{c}}$ which contains all those vectors $\boldsymbol{c}^{\prime}$ that have arrived (in other words, have been projected) into the point $\boldsymbol{c} \in \Omega$. Then we observe that the state $\boldsymbol{c}$ furnishes the minimum of the function $G$ on the set $\boldsymbol{U}_{\boldsymbol{c}}$. If a state $\boldsymbol{c}^{\prime} \in \boldsymbol{U}_{\boldsymbol{c}}$, and if it deviates small enough from the state $\boldsymbol{c}$ so that the linear approximation is valid, then $\boldsymbol{c}^{\prime}$ belongs to the affine hyperplane

$$
\Gamma_{\boldsymbol{c}}=\boldsymbol{c}+\operatorname{ker} M_{\boldsymbol{c}}^{*}, \boldsymbol{c} \in \Omega .
$$

This hyperplane actually participates in the condition (35). The consideration was entitled 'thermodynamic' [24] because it describes the states $\boldsymbol{c} \in \boldsymbol{\Omega}$ as points of minimum of the function $G$ over the corresponding hyperplanes (44).

\section{Corrections}

\subsection{Preliminary discussion}

The thermodynamic projector is needed to induce the dynamics on a given manifold in such a way that the dissipation inequality (33) holds. Coming back to the issue of constructing corrections, we should stress that the projector participating in the invariance condition (36) is arbitrary. It is convenient to make use of this point: When Eq. (36) is solved iteratively, the projector may be kept non-thermodynamic unless the induced dynamics is explicitly needed.

Let us assume that we have chosen the initial manifold, $\boldsymbol{\Omega}_{0}$, together with the associated projector $\boldsymbol{P}_{0}$, as the first approximation to the desired manifold of reduced description. Though the choice of the initial approximation $\boldsymbol{\Omega}_{0}$ depends on the specific problem, it is 
often reasonable to consider quasi-equilibrium or quasi steady-state approximations. In most cases, the manifold $\Omega_{0}$ is not an invariant manifold. This means that $\Omega_{0}$ does not satisfy the invariance condition (36):

$$
\boldsymbol{\Delta}_{0}=\left[1-\boldsymbol{P}_{0}\right] \boldsymbol{J}\left(\boldsymbol{c}_{0}\right) \neq 0, \text { for some } \boldsymbol{c}_{0} \in \boldsymbol{\Omega}_{0}
$$

Therefore, we seek a correction $\boldsymbol{c}_{1}=\boldsymbol{c}_{0}+\delta \boldsymbol{c}$. Substituting $\boldsymbol{P}=\boldsymbol{P}_{0}$ and $\boldsymbol{c}=\boldsymbol{c}_{0}+\delta \boldsymbol{c}$ into the invariance equation (36), and after the linearization in $\delta \boldsymbol{c}$, we derive the following linear equation:

$$
\left[1-\boldsymbol{P}_{0}\right]\left[\boldsymbol{J}\left(\boldsymbol{c}_{0}\right)+\boldsymbol{L}_{\boldsymbol{c}_{0}} \delta \boldsymbol{c}\right]=0
$$

where $\boldsymbol{L}_{c_{0}}$ is the matrix of first derivatives of the vector function $\boldsymbol{J}$, computed in the state $\boldsymbol{c}_{0} \in \boldsymbol{\Omega}_{0}$. The system of linear algebraic equations (46) should be supplied with the additional condition.

$$
\boldsymbol{P}_{0} \delta \boldsymbol{c}=0
$$

In order to illustrate the nature of the Eq. (46), let us consider the case of linear manifolds for linear systems. Let a linear evolution equation is given in the finite-dimensional real space: $\dot{\boldsymbol{c}}=\boldsymbol{L} \boldsymbol{c}$, where $\boldsymbol{L}$ is negatively definite symmetric matrix with a simple spectrum. Let us further assume the quadratic Lyapunov function, $G(\boldsymbol{c})=(\boldsymbol{c}, \boldsymbol{c})$. The manifolds we consider are lines, $\boldsymbol{l}(a)=a \boldsymbol{e}$, where $\boldsymbol{e}$ is the unit vector, and $a$ is a scalar. The invariance equation for such manifolds reads: $\boldsymbol{e}(\boldsymbol{e}, \boldsymbol{L} \boldsymbol{e})-\boldsymbol{L} \boldsymbol{e}=0$, and is simply a form of the eigenvalue problem for the operator $\boldsymbol{L}$. Solutions to the latter equation are eigenvectors $\boldsymbol{e}_{i}$, corresponding to eigenvalues $\lambda_{i}$.

Assume that we have chosen a line, $\boldsymbol{l}_{0}=a \boldsymbol{e}_{0}$, defined by the unit vector $\boldsymbol{e}_{0}$, and that $e_{0}$ is not an eigenvector of $\boldsymbol{L}$. We seek another line, $\boldsymbol{l}_{1}=a \boldsymbol{e}_{1}$, where $\boldsymbol{e}_{1}$ is another unit vector, $\boldsymbol{e}_{1}=\boldsymbol{y}_{1} /\left\|\boldsymbol{y}_{1}\right\|, \boldsymbol{y}_{1}=\boldsymbol{e}_{0}+\delta \boldsymbol{y}$. The additional condition (47) now reads: $\left(\delta \boldsymbol{y}, \boldsymbol{e}_{0}\right)=0$. Then the Eq. (46) becomes $\left[1-\boldsymbol{e}_{0}\left(\boldsymbol{e}_{0}, \cdot\right)\right] L\left[\boldsymbol{e}_{0}+\delta \boldsymbol{y}\right]=0$. Subject to the additional condition, the unique solution is as follows: $\boldsymbol{e}_{0}+\delta \boldsymbol{y}=\left(\boldsymbol{e}_{0}, \boldsymbol{L}^{-1} \boldsymbol{e}_{0}\right)^{-1} \boldsymbol{L}^{-1} \boldsymbol{e}_{0}$. Rewriting the latter expression in the eigen-basis of $\boldsymbol{L}$, we have: $\boldsymbol{e}_{0}+\delta \boldsymbol{y} \propto \sum_{i} \lambda_{i}^{-1} \boldsymbol{e}_{i}\left(\boldsymbol{e}_{i}, \boldsymbol{e}_{0}\right)$. The leading term in this sum corresponds to the eigenvalue with the minimal absolute value. The example indicates that the method of linearization (46) seeks the direction of the slowest relaxation. For this reason, the method (46) can be recognized as the basis of an iterative method for constructing the manifolds of slow motions.

For the nonlinear systems, the matrix $\boldsymbol{L}_{c_{0}}$ in the Eq. (46) depends nontrivially on $\boldsymbol{c}_{0}$. In this case the system (46) requires a further specification which will be done now. 


\subsection{Symmetric linearization}

The invariance condition (36) supports a lot of invariant manifolds, and not all of them are relevant to the reduced description (for example, any individual trajectory is itself an invariant manifold). This should be carefully taken into account when deriving a relevant equation for the correction in the states of the initial manifold $\Omega_{0}$ which are located far from equilibrium. This point concerns the procedure of the linearization of the vector field $\boldsymbol{J}$, appearing in the equation (46). We shall return to the explicit form of the Marcelin-De Donder kinetic function (11). Let $\boldsymbol{c}$ is an arbitrary fixed element of the phase space. The linearization of the vector function $\boldsymbol{J}(12)$ about $\boldsymbol{c}$ may be written $\boldsymbol{J}(\boldsymbol{c}+\delta \boldsymbol{c}) \approx \boldsymbol{J}(\boldsymbol{c})+\boldsymbol{L}_{\boldsymbol{c}} \delta \boldsymbol{c}$ where the linear operator $\boldsymbol{L}_{\boldsymbol{c}}$ acts as follows:

$$
\boldsymbol{L}_{\boldsymbol{c}} \boldsymbol{x}=\sum_{s=1}^{r} \boldsymbol{\gamma}_{s}\left[W_{s}^{+}(\boldsymbol{c})\left(\boldsymbol{\alpha}_{s}, \boldsymbol{H}_{\boldsymbol{c}} \boldsymbol{x}\right)-W_{s}^{-}(\boldsymbol{c})\left(\boldsymbol{\beta}_{s}, \boldsymbol{H}_{\boldsymbol{c}} \boldsymbol{x}\right)\right] .
$$

Here $\boldsymbol{H}_{\boldsymbol{c}}$ is the matrix of second derivatives of the function $G$ in the state $\boldsymbol{c}$ [see Eq. (7)]. The matrix $\boldsymbol{L}_{\boldsymbol{c}}$ in the Eq. (48) can be decomposed as follows:

$$
L_{c}=L_{c}^{\prime}+L_{c}^{\prime \prime}
$$

Matrices $\boldsymbol{L}_{\boldsymbol{c}}^{\prime}$ and $\boldsymbol{L}_{\boldsymbol{c}}^{\prime \prime}$ act as follows:

$$
\begin{aligned}
& \boldsymbol{L}_{\boldsymbol{c}}^{\prime} \boldsymbol{x}=-\frac{1}{2} \sum_{s=1}^{r}\left[W_{s}^{+}(\boldsymbol{c})+W_{s}^{-}(\boldsymbol{c})\right] \boldsymbol{\gamma}_{s}\left(\boldsymbol{\gamma}_{s}, \boldsymbol{H}_{\boldsymbol{c}} \boldsymbol{x}\right) \\
& \boldsymbol{L}_{\boldsymbol{c}}^{\prime \prime} \boldsymbol{x}=\frac{1}{2} \sum_{s=1}^{r}\left[W_{s}^{+}(\boldsymbol{c})-W_{s}^{-}(\boldsymbol{c})\right] \boldsymbol{\gamma}_{s}\left(\boldsymbol{\alpha}_{s}+\boldsymbol{\beta}_{s}, \boldsymbol{H}_{\boldsymbol{c}} \boldsymbol{x}\right) .
\end{aligned}
$$

Some features of this decomposition are best seen when we use the thermodynamic scalar product (28): The following properties of the matrix $\boldsymbol{L}_{\boldsymbol{c}}^{\prime}$ are verified immediately:

(i) The matrix $\boldsymbol{L}_{\boldsymbol{c}}^{\prime}$ is symmetric in the scalar product (28):

$$
\left\langle\boldsymbol{x}, \boldsymbol{L}_{\boldsymbol{c}}^{\prime} \boldsymbol{y}\right\rangle=\left\langle\boldsymbol{y}, \boldsymbol{L}_{\boldsymbol{c}}^{\prime} \boldsymbol{x}\right\rangle
$$

(ii) The matrix $\boldsymbol{L}_{\boldsymbol{c}}^{\prime}$ is nonpositive definite in the scalar product (28):

$$
\left\langle\boldsymbol{x}, \boldsymbol{L}_{\boldsymbol{c}}^{\prime} \boldsymbol{x}\right\rangle \leq 0
$$

(iii) The null space of the matrix $\boldsymbol{L}_{\boldsymbol{c}}^{\prime}$ is the linear envelope of the vectors $\boldsymbol{H}_{\boldsymbol{c}}^{-1} \boldsymbol{b}_{i}$ representing the complete system of conservation laws:

$$
\operatorname{ker} \boldsymbol{L}_{\boldsymbol{c}}^{\prime}=\operatorname{Lin}\left\{\boldsymbol{H}_{\boldsymbol{c}}^{-1} \boldsymbol{b}_{i}, i=1, \ldots, l\right\}
$$


(iv) If $\boldsymbol{c}=\boldsymbol{c}^{\mathrm{eq}}$, then $W_{s}^{+}\left(\boldsymbol{c}^{\mathrm{eq}}\right)=W_{s}^{-}\left(\boldsymbol{c}^{\mathrm{eq}}\right)$, and

$$
\boldsymbol{L}_{\boldsymbol{C}^{\mathrm{eq}}}^{\prime}=\boldsymbol{L}_{\boldsymbol{C}^{\mathrm{eq}}}
$$

Thus, the decomposition Eq. (49) splits the matrix $\boldsymbol{L}_{\boldsymbol{c}}$ in two parts: one part, Eq. (50) is symmetric and nonpositive definite, while the other part, Eq. (51), vanishes in the equilibrium. The decomposition Eq. (49) explicitly takes into account the Marcelin-De Donder form of the kinetic function. For other dissipative systems, the decomposition (49) is possible as soon as the relevant kinetic operator is written in a gain-loss form [for instance, this is straightforward for the Boltzmann collision operator].

In the sequel, we shall make use of the properties of the operator $\boldsymbol{L}_{\boldsymbol{c}}^{\prime}$ (50) for constructing the dynamic correction by extending the picture of the decomposition of motions.

\subsection{Decomposition of motions: Kinetics}

The assumption about the existence of the decomposition of motions near the manifold of reduced description $\Omega$ has led to the thermodynamic specifications of the states $\boldsymbol{c} \in$ $\boldsymbol{\Omega}$. This was accomplished in the section 4.2 , where the thermodynamic projector was backed by an appropriate variational formulation, and this helped us to establish the induced dynamics consistent with the dissipation property. Another important feature of the decomposition of motions is that the states $\boldsymbol{c} \in \boldsymbol{\Omega}$ can be specified kinetically. Indeed, let us do it again as if the decomposition of motions were valid in the neighborhood of the manifold $\Omega$, and let us 'freeze' the slow dynamics along the $\Omega$, focusing on the fast process of relaxation towards a state $\boldsymbol{c} \in \boldsymbol{\Omega}$. From the thermodynamic perspective, fast motions take place on the affine hyperplane $\boldsymbol{c}+\delta \boldsymbol{c} \in \Gamma_{\boldsymbol{c}_{0}}$, where $\Gamma_{\boldsymbol{c}_{0}}$ is given by Eq. (44). From the kinetic perspective, fast motions on this hyperplane should be treated as a relaxation equation, equipped with the quadratic Lyapunov function $\delta G=\langle\delta \boldsymbol{c}, \delta \boldsymbol{c}\rangle$, Furthermore, we require that the linear operator of this evolution equation should respect Onsager's symmetry requirements (selfadjointness with respect to the entropic scalar product). This latter crucial requirement describes fast motions under the frozen slow evolution in the similar way, as all the motions near the equilibrium.

Let us consider now the manifold $\Omega_{0}$ which is not the invariant manifold of the reduced description but, by our assumption, is located close to it. Consider a state $\boldsymbol{c}_{0} \in \boldsymbol{\Omega}_{0}$, and the states $\boldsymbol{c}_{0}+\delta \boldsymbol{c}$ close to it. Further, let us consider an equation

$$
\dot{\delta \boldsymbol{c}}=\boldsymbol{L}_{\boldsymbol{c}_{0}}^{\prime} \delta \boldsymbol{c}
$$

Due to the properties of the operator $\boldsymbol{L}_{\boldsymbol{c}_{0}}^{\prime}(50)$, this equation can be regarded as a model of the assumed true relaxation equation near the true manifold of the reduced description. For this reason, we shall use the symmetric operator $\boldsymbol{L}_{\boldsymbol{c}}^{\prime}(50)$ instead of the linear operator $\boldsymbol{L}_{\boldsymbol{c}}$ when constructing the corrections. 


\subsection{Symmetric iteration}

Let the manifold $\boldsymbol{\Omega}_{0}$ and the corresponding projector $\boldsymbol{P}_{0}$ are the initial approximation to the invariant manifold of the reduced description. The dynamic correction $\boldsymbol{c}_{1}=\boldsymbol{c}_{0}+\delta \boldsymbol{c}$ is found upon solving the following system of linear algebraic equations:

$$
\left[1-\boldsymbol{P}_{0}\right]\left[\boldsymbol{J}\left(\boldsymbol{c}_{0}\right)+\boldsymbol{L}_{\boldsymbol{c}_{0}}^{\prime} \delta \boldsymbol{c}\right]=0, \boldsymbol{P}_{0} \delta \boldsymbol{c}=0
$$

Here $\boldsymbol{L}_{\boldsymbol{c}_{0}}^{\prime}$ is the matrix (50) taken in the states on the manifold $\boldsymbol{\Omega}_{0}$. An important technical point here is that the linear system (57) always has the unique solution for any choice of the manifold $\boldsymbol{\Omega}$. This point is crucial since it guarantees the opportunity of carrying out the correction process for arbitrary number of steps.

\section{The method of invariant manifold}

We shall now combine together the two procedures discussed above. The resulting method of invariant manifold intends to seek iteratively the reduced description, starting with an initial approximation.

(i). Initialization. In order to start the procedure, it is required to choose the initial manifold $\boldsymbol{\Omega}_{0}$, and to derive corresponding thermodynamic projector $\boldsymbol{P}_{0}$. In the majority of cases, initial manifolds are available in two different ways. The first case are the quasiequilibrium manifolds described in the section 2.3. The macroscopic parameters are $M_{i}=$ $c_{i}=\left(\boldsymbol{m}_{i}, \boldsymbol{c}\right)$, where $\boldsymbol{m}_{i}$ is the unit vector corresponding to the specie $A_{i}$. The quasiequilibrium manifold, $\boldsymbol{c}_{0}\left(M_{1}, \ldots, M_{k}, B_{1}, \ldots, B_{l}\right)$, compatible with the conservation laws, is the solution to the variational problem:

$$
\begin{aligned}
& G \rightarrow \min ,\left(\boldsymbol{m}_{i}, \boldsymbol{c}\right)=c_{i}, i=1, \ldots, k, \\
& \left(\boldsymbol{b}_{j}, \boldsymbol{c}\right)=B_{j}, j=1, \ldots, l \text {. }
\end{aligned}
$$

In the case of quasi-equilibrium approximation, the corresponding thermodynamic projector can be written most straightforwardly in terms of the variables $M_{i}$ :

$$
\boldsymbol{P}_{0} \boldsymbol{x}=\sum_{i=1}^{k} \frac{\partial \boldsymbol{c}_{0}}{\partial c_{i}}\left(\boldsymbol{m}_{i}, \boldsymbol{x}\right)+\sum_{i=1}^{l} \frac{\partial \boldsymbol{c}_{0}}{\partial B_{i}}\left(\boldsymbol{b}_{i}, \boldsymbol{x}\right) .
$$

For quasi-equilibrium manifolds, a reparameterization with the set $(42),(43)$ is not necessary $([24] ;[28])$.

The second source of initial approximations are quasi-stationary manifolds (section 2.5). Unlike the quasi-equilibrium case, the quasi-stationary manifolds must be reparameterized in order to construct the thermodynamic projector. 
(ii). Corrections. Iterations are organized in accord with the rule: If $\boldsymbol{c}_{m}$ is the $m$ th approximation to the invariant manifold, then the correction $\boldsymbol{c}_{m+1}=\boldsymbol{c}_{m}+\delta \boldsymbol{c}$ is found from the linear algebraic equations,

$$
\begin{aligned}
{\left[1-\boldsymbol{P}_{m}\right]\left(\boldsymbol{J}\left(\boldsymbol{c}_{m}\right)+\boldsymbol{L}_{\boldsymbol{c}_{m}}^{\prime} \delta \boldsymbol{c}\right) } & =0 \\
\boldsymbol{P}_{m} \delta \boldsymbol{c} & =0 .
\end{aligned}
$$

Here $\boldsymbol{L}_{\boldsymbol{c}_{m}}^{\prime}$ is the symmetric matrix (50) evaluated at the $m$ th approximation. The projector $\boldsymbol{P}_{m}$ is not obligatory thermodynamic at that step, and it is taken as follows:

$$
\boldsymbol{P}_{m} \boldsymbol{x}=\sum_{i=1}^{k} \frac{\partial \boldsymbol{c}_{m}}{\partial c_{i}}\left(\boldsymbol{m}_{i}, \boldsymbol{x}\right)+\sum_{i=1}^{l} \frac{\partial \boldsymbol{c}_{m}}{\partial B_{i}}\left(\boldsymbol{b}_{i}, \boldsymbol{x}\right) .
$$

(iii). Dynamics. Dynamics on the $m$ th manifold is obtained with the thermodynamic re-parameterization.

In the next section we shall illustrate how this all works.

\section{Illustration: Two-step catalytic reaction}

Here we consider a two-step four-component reaction with one catalyst $A_{2}$ :

$$
A_{1}+A_{2} \rightleftharpoons A_{3} \rightleftharpoons A_{2}+A_{4}
$$

We assume the Lyapunov function of the form (9), $G=\sum_{i=1}^{4} c_{i}\left[\ln \left(c_{i} / c_{i}^{\mathrm{eq}}\right)-1\right]$. The kinetic equation for the four-component vector of concentrations, $\boldsymbol{c}=\left(c_{1}, c_{2}, c_{3}, c_{4}\right)$, has the form

$$
\dot{\boldsymbol{c}}=\gamma_{1} W_{1}+\gamma_{2} W_{2}
$$

Here $\gamma_{1,2}$ are stoichiometric vectors,

$$
\gamma_{1}=(-1,-1,1,0), \gamma_{2}=(0,1,-1,1)
$$

while functions $W_{1,2}$ are reaction rates:

$$
W_{1}=k_{1}^{+} c_{1} c_{2}-k_{1}^{-} c_{3}, W_{2}=k_{2}^{+} c_{3}-k_{2}^{-} c_{2} c_{4}
$$

Here $k_{1,2}^{ \pm}$are reaction rate constants. The system under consideration has two conservation laws,

$$
c_{1}+c_{3}+c_{4}=B_{1}, c_{2}+c_{3}=B_{2},
$$


or $\left(\boldsymbol{b}_{1,2}, \boldsymbol{c}\right)=B_{1,2}$, where $\boldsymbol{b}_{1}=(1,0,1,1)$ and $\boldsymbol{b}_{2}=(0,1,1,0)$. The nonlinear system $(64)$ is effectively two-dimensional, and we consider a one-dimensional reduced description.

We have chosen the concentration of the specie $A_{1}$ as the variable of reduced description: $M=c_{1}$, and $c_{1}=(\boldsymbol{m}, \boldsymbol{c})$, where $\boldsymbol{m}=(1,0,0,0)$. The initial manifold $\boldsymbol{c}_{0}(M)$ was taken as the quasi-equilibrium approximation, i.e. the vector function $\boldsymbol{c}_{0}$ is the solution to the problem:

$$
G \rightarrow \min \text { for }(\boldsymbol{m}, \boldsymbol{c})=c_{1},\left(\boldsymbol{b}_{1}, \boldsymbol{c}\right)=B_{1},\left(\boldsymbol{b}_{2}, \boldsymbol{c}\right)=B_{2}
$$

The solution to the problem (68) reads:

$$
\begin{aligned}
c_{01} & =c_{1}, \\
c_{02} & =B_{2}-\phi\left(c_{1}\right), \\
c_{03} & =\phi\left(c_{1}\right), \\
c_{04} & =B_{1}-c_{1}-\phi\left(c_{1}\right), \\
\phi(M) & =A\left(c_{1}\right)-\sqrt{A^{2}\left(c_{1}\right)-B_{2}\left(B_{1}-c_{1}\right)}, \\
A\left(c_{1}\right) & =\frac{B_{2}\left(B_{1}-c_{1}^{\mathrm{eq}}\right)+c_{3}^{\mathrm{eq}}\left(c_{1}^{\mathrm{eq}}+c_{3}^{\mathrm{eq}}-c_{1}\right)}{2 c_{3}^{\mathrm{eq}}} .
\end{aligned}
$$

The thermodynamic projector associated with the manifold (69) reads:

$$
\boldsymbol{P}_{0} \boldsymbol{x}=\frac{\partial \boldsymbol{c}_{0}}{\partial c_{1}}(\boldsymbol{m}, \boldsymbol{x})+\frac{\partial \boldsymbol{c}_{0}}{\partial B_{1}}\left(\boldsymbol{b}_{1}, \boldsymbol{x}\right)+\frac{\partial \boldsymbol{c}_{0}}{\partial B_{2}}\left(\boldsymbol{b}_{2}, \boldsymbol{x}\right)
$$

Computing $\boldsymbol{\Delta}_{0}=\left[1-\boldsymbol{P}_{0}\right] \boldsymbol{J}\left(\boldsymbol{c}_{0}\right)$ we find that the inequality (45) takes place, and thus the manifold $\boldsymbol{c}_{0}$ is not invariant. The first correction, $\boldsymbol{c}_{1}=\boldsymbol{c}_{0}+\delta \boldsymbol{c}$, is found from the linear algebraic system (60)

$$
\begin{aligned}
\left(1-\boldsymbol{P}_{0}\right) \boldsymbol{L}_{0}^{\prime} \delta \boldsymbol{c} & =-\left[1-\boldsymbol{P}_{0}\right] \boldsymbol{J}\left(\boldsymbol{c}_{0}\right), \\
\delta c_{1} & =0 \\
\delta c_{1}+\delta c_{3}+\delta c_{4} & =0 \\
\delta c_{3}+\delta c_{2} & =0
\end{aligned}
$$

where the symmetric $4 \times 4$ matrix $\boldsymbol{L}_{0}^{\prime}$ has the form (we write 0 instead of $\boldsymbol{c}_{0}$ in the subscript in order to simplify notations):

$$
L_{0, k l}^{\prime}=-\gamma_{1 k} \frac{W_{1}^{+}\left(\boldsymbol{c}_{0}\right)+W_{1}^{-}\left(\boldsymbol{c}_{0}\right)}{2} \frac{\gamma_{1 l}}{c_{0 l}}-\gamma_{2 k} \frac{W_{2}^{+}\left(\boldsymbol{c}_{0}\right)+W_{2}^{-}\left(\boldsymbol{c}_{0}\right)}{2} \frac{\gamma_{2 l}}{c_{0 l}}
$$

The explicit solution $\boldsymbol{c}_{1}\left(c_{1}, B_{1}, B_{2}\right)$ to the linear system (71) is easily found, and we do not reproduce it here. The process was iterated. On the $k+1$ iteration, the following 
projector $\boldsymbol{P}_{k}$ was used:

$$
\boldsymbol{P}_{k} \boldsymbol{x}=\frac{\partial \boldsymbol{c}_{k}}{\partial c_{1}}(\boldsymbol{m}, \boldsymbol{x})+\frac{\partial \boldsymbol{c}_{k}}{\partial B_{1}}\left(\boldsymbol{b}_{1}, \boldsymbol{x}\right)+\frac{\partial \boldsymbol{c}_{k}}{\partial B_{2}}\left(\boldsymbol{b}_{2}, \boldsymbol{x}\right)
$$

Notice that projector $\boldsymbol{P}_{k}(74)$ is the thermodynamic projector only if $k=0$. As we have already mentioned it above, in the process of finding the corrections to the manifold, the non-thermodynamic projectors are allowed. The linear equation at the $k+1$ iteration is thus obtained by replacing $\boldsymbol{c}_{0}, \boldsymbol{P}_{0}$, and $\boldsymbol{L}_{0}^{\prime}$ with $\boldsymbol{c}_{k}, \boldsymbol{P}_{k}$, and $\boldsymbol{L}_{k}^{\prime}$ in all the entries of the Eqs. (71) and (73).

Once the manifold $\boldsymbol{c}_{k}$ was obtained on the $k$ th iteration, we derived the corresponding dynamics by introducing the thermodynamic parameterization (and the corresponding thermodynamic projector) with the help of the function (42). The resulting dynamic equation for the variable $c_{1}$ in the $k$ th approximation has the form:

$$
\left(\left.\boldsymbol{\nabla} G\right|_{\boldsymbol{c}_{k}}, \partial \boldsymbol{c}_{k} / \partial c_{1}\right) \dot{c_{1}}=\left(\left.\nabla G\right|_{\boldsymbol{c}_{k}}, \boldsymbol{J}\left(\boldsymbol{c}_{k}\right)\right)
$$

Here $\left[\left.\nabla G\right|_{\boldsymbol{c}_{k}}\right]_{i}=\ln \left[c_{k i} / c_{i}^{\mathrm{eq}}\right]$.

Analytic results were compared with the results of the numerical integration. The following set of parameters was used:

$$
\begin{array}{r}
k_{1}^{+}=1.0, \quad k_{1}^{-}=0.5, k_{2}^{+}=0.4, \quad k_{2}^{-}=1.0 \\
c_{1}^{\text {eq }}=0.5, \quad c_{2}^{\text {eq }}=0.1, \\
c_{3}^{\text {eq }}=0.1, \quad c_{4}^{\text {eq }}=0.4 \\
B_{1}=1.0, \quad B_{2}=0.2 .
\end{array}
$$

Direct numerical integration of the system has demonstrated that the manifold $c_{3}=c_{3}^{\text {eq }}$ in the plane $\left(c_{1}, c_{3}\right)$ attracts all individual trajectories. Thus, the reduced description in this example should extract this manifold.

Fig. 1 demonstrates the quasi-equilibrium manifold (69) and the first two corrections found analytically. It should be stressed that we spend no special effort on the construction of the initial approximation, that is, of the quasi-equilibrium manifold, have not used any information about the Jacobian field (unlike, for example, the ILDM or CSP methods discussed above) etc. It is therefore not surprising that in this way chosen initial quasiequilibrium approximation is in a rather poor agreement with the reduced description. However, it should be appreciated that the further corrections rapidly improve the situation while no small parameter considerations were used. This confirms our expectation of the advantage of using the iteration methods in comparison to methods based on a small parameter expansions for model reduction problems. 


\section{Relaxation methods}

Relaxation method is an alternative to the Newton iteration method described in section 5. It is a one-dimensional Galerkin approximation for the linearized invariance equation $(46,47)$. We shall solve the invariance equation $(46,47)$ (or symmetric invariance equation $(60,61)$ in projection on the defect of invariance (45) $\boldsymbol{\Delta}=\left[1-\boldsymbol{P}_{\boldsymbol{c}}\right] \boldsymbol{J}(\boldsymbol{c})$.

Let $\Omega_{0}$ be the initial approximation to the invariant manifold, and we seek the first correction, $\boldsymbol{c}_{1}=\boldsymbol{c}_{0}+\tau_{1}\left(\boldsymbol{c}_{0}\right) \boldsymbol{\Delta}\left(\boldsymbol{c}_{0}\right)$, where function $\tau\left(\boldsymbol{c}_{0}\right)$ has a dimension of the time, and is found from the condition that the linearized vector field attached to the points of the new manifold is orthogonal to the initial defect,

$$
\left\langle\boldsymbol{\Delta}\left(\boldsymbol{c}_{0}\right),\left(1-\boldsymbol{P}_{\boldsymbol{c}_{0}}\right)\left[\boldsymbol{J}\left(\boldsymbol{c}_{0}\right)+\tau_{1}\left(\boldsymbol{c}_{0}\right)\left(D_{\boldsymbol{c}} \boldsymbol{J}\right) \boldsymbol{c}_{0} \boldsymbol{\Delta}_{\boldsymbol{c}_{0}}\right]\right\rangle_{\boldsymbol{c}_{0}}=0
$$

Explicitly,

$$
\tau_{1}\left(\boldsymbol{c}_{0}\right)=-\frac{\left\langle\boldsymbol{\Delta}_{\boldsymbol{c}_{0}}, \boldsymbol{\Delta}_{\boldsymbol{c}_{0}}\right\rangle \boldsymbol{c}_{0}}{\left\langle\boldsymbol{\Delta}_{\boldsymbol{c}_{0}},\left(D_{\boldsymbol{c}} \boldsymbol{J}\right) \boldsymbol{c}_{0} \boldsymbol{\Delta}_{\boldsymbol{c}_{0}}\right\rangle \boldsymbol{c}_{0}}
$$

Further steps $\tau_{k}(\boldsymbol{c})$ are found in the same way. It is clear from the latter equations that the step of the relaxation method is equivalent to the Galerkin approximation for solving the step of the Newton method. Actually, the relaxation method was first introduced in these terms in [52]. An advantage of equation (77) is the explicit form of the size of the steps $\tau_{k}(\boldsymbol{c})$. This method was successfully applied to the Fokker-Plank equation [52].

\section{Method of invariant manifold without a priori parameterization}

Formally, the method of invariant manifold does not require a global parameterization of the manifolds. However, in most of the cases, one makes use of a priori defined "macroscopic" variables $M$. This is motivated by the choice of quasi-equilibrium initial approximations.

Let a manifold $\Omega$ be defined in the phase space of the system, its tangent space in the point $\boldsymbol{c}$ be $T_{\boldsymbol{c}} \Omega$. How to define the projector of the whole concentrations space onto $T_{\boldsymbol{c}} \boldsymbol{\Omega}$ without using any a priori parameterization of $\Omega$ ?

The basis of the answer to this question is the condition of thermodynamicity (35). Let us denote $E$ as the concentration space, and consider the problem of the choice of the projector in the quadratic approximation to the thermodynamic potential $G$ :

$$
G_{\mathrm{q}}=\left(\boldsymbol{g}, \boldsymbol{H}_{\boldsymbol{c}} \Delta \boldsymbol{c}\right)+\frac{1}{2}\left(\Delta \boldsymbol{c}, \boldsymbol{H}_{\boldsymbol{c}} \Delta \boldsymbol{c}\right)=\langle\boldsymbol{g}, \Delta \boldsymbol{c}\rangle+\frac{1}{2}\langle\Delta \boldsymbol{c}, \Delta \boldsymbol{c}\rangle
$$


where $\boldsymbol{H}_{\boldsymbol{c}}$ is the matrix of the second-order derivatives of $G(7), \boldsymbol{g}=\boldsymbol{H}_{\boldsymbol{c}}^{-1} \boldsymbol{\nabla} G, \Delta \boldsymbol{c}$ is the deviation of the concentration vector from the expansion point.

Let a linear subspace $T$ be given in the concentrations space $E$. Problem: For every $\Delta \boldsymbol{c}+T$, and for every $\boldsymbol{g} \in E$, define a subspace $L_{\Delta \boldsymbol{c}}$ such that: (i) $L_{\Delta \boldsymbol{c}}$ is a complement of $T$ in $E$ :

$$
L_{\Delta \boldsymbol{c}}+T=E, L_{\Delta \boldsymbol{c}} \cap T=\{\mathbf{0}\}
$$

(ii) $\Delta \boldsymbol{c}$ is the point of minimum of $G_{\mathrm{q}}$ on $L_{\Delta \boldsymbol{c}}+\Delta \boldsymbol{c}$ :

$$
\Delta \boldsymbol{c}=\arg \min _{\boldsymbol{x}-\Delta \boldsymbol{c} \in L_{\Delta} \boldsymbol{c}} G_{\mathrm{q}}(\boldsymbol{x})
$$

Besides (i) and (ii), we also impose the requirement of a maximal smoothness (analyticity) on $L_{\Delta \boldsymbol{c}}$ as a function of $\boldsymbol{g}$ and $\Delta \boldsymbol{c}$. Requirement (79) implies that $\Delta \boldsymbol{c}$ is the quasi-equilibrium point for the given $L_{\Delta \boldsymbol{c}}$, while the problem in a whole is the inverse quasi-equilibrium problem: We construct $L_{\Delta \boldsymbol{c}}$ such that $T$ will be the quasi-equilibrium manifold. Then subspaces $L_{\Delta} \boldsymbol{c}$ will actually be the kernels of the quasi-equilibrium projector.

Let $\boldsymbol{f}_{1}, \ldots, \boldsymbol{f}_{k}$ be the orthonormalized with respect to $\langle\cdot, \cdot\rangle$ scalar product basis of $T$, vector $\boldsymbol{h}$ be orthogonal to $T,\langle\boldsymbol{h}, \boldsymbol{h}\rangle=1, \boldsymbol{g}=\alpha \boldsymbol{f}_{1}+\beta \boldsymbol{h}$. Condition (79) implies that the vector $\boldsymbol{\nabla} G$ is orthogonal to $L_{\Delta \boldsymbol{c}}$ in the point $\Delta \boldsymbol{c}$.

Let us first consider the case $\beta=0$. The requirement of analyticity of $L_{\Delta} \boldsymbol{c}$ as the function of $\alpha$ and $\Delta \boldsymbol{c}$ implies $L_{\Delta \boldsymbol{c}}=L_{\mathbf{0}}+o(1)$, where $L_{\mathbf{0}}=T^{\perp}$ is the orthogonal completement of $T$ with respect to scalar product $\langle\cdot, \cdot\rangle$. The constant solution, $L_{\Delta \boldsymbol{c}} \equiv L_{\mathbf{0}}$ also satisfies (79). Let us fix $\alpha \neq 0$, and extend this latter solution to $\beta \neq 0$. With this, we obtain a basis, $\boldsymbol{l}_{1}, \ldots, \boldsymbol{l}_{n-k}$. Here is the simplest construction of this basis:

$$
\boldsymbol{l}_{1}=\frac{\beta \boldsymbol{f}_{1}-\left(\alpha+\Delta c_{1}\right) \boldsymbol{h}}{\left(\beta^{2}+\left(\alpha+\Delta c_{1}\right)^{2}\right)^{1 / 2}}
$$

where $\Delta c_{1}=\left\langle\Delta \boldsymbol{c}, \boldsymbol{f}_{1}\right\rangle$ is the first component in the expansion, $\Delta \boldsymbol{c}=\sum_{i} \Delta c_{i} \boldsymbol{f}_{i}$. The rest of the basis elements, $\boldsymbol{l}_{2}, \ldots, \boldsymbol{l}_{n-k}$ form the orthogonal completement of $T \oplus(\boldsymbol{h})$ with respect to scalar product $\langle\cdot, \cdot\rangle,(\boldsymbol{h})$ is the line spanned by $\boldsymbol{h}$.

Dependence $L_{\Delta \boldsymbol{c}}(80)$ on $\Delta \boldsymbol{c}, \alpha$ and $\beta$ is singular: At $\alpha+\Delta c_{1}$, vector $\boldsymbol{l}_{1} \in T$, and then $L_{\Delta \boldsymbol{c}}$ is not the completement of $T$ in $E$ anymore. For $\alpha \neq 0$, dependence $L_{\Delta \boldsymbol{c}}$ gives one of the solutions to the inverse quasi-equilibrium problem in the neighborhood of zero in $T$. We are interested only in the limit,

$$
\lim _{\Delta \boldsymbol{c} \rightarrow \mathbf{0}} L_{\Delta \boldsymbol{c}}=\operatorname{Lin}\left\{\frac{\beta \boldsymbol{f}_{1}-\alpha \boldsymbol{h}}{\sqrt{\alpha^{2}+\beta^{2}}}, \boldsymbol{l}_{2}, \ldots, \boldsymbol{l}_{n-k}\right\} .
$$


Finally, let us define now the projector $\boldsymbol{P}_{\boldsymbol{c}}$ of the space $E$ onto $T_{\boldsymbol{c}} \boldsymbol{\Omega}$. If $\boldsymbol{H}_{\boldsymbol{c}}^{-1} \boldsymbol{\nabla} G \in T_{\boldsymbol{c}} \boldsymbol{\Omega}$, then $\boldsymbol{P}_{\boldsymbol{c}}$ is the orthogonal projector with respect to the scalar product $\langle\cdot, \cdot\rangle$ :

$$
\boldsymbol{P}_{\boldsymbol{c}} \boldsymbol{z}=\sum_{i=1}^{k} \boldsymbol{f}_{i}\left\langle\boldsymbol{f}_{i}, \boldsymbol{z}\right\rangle
$$

If $\boldsymbol{H}_{\boldsymbol{c}}^{-1} \nabla G \notin T_{\boldsymbol{c}} \boldsymbol{\Omega}$, then, according to Eq. (81),

$$
\boldsymbol{P}_{\boldsymbol{c}} \boldsymbol{z}=\frac{\left\langle\boldsymbol{f}_{1}, \boldsymbol{z}\right\rangle-\left\langle\boldsymbol{l}_{1}, \boldsymbol{z}\right\rangle\left\langle\boldsymbol{f}_{1}, \boldsymbol{l}_{1}\right\rangle}{1-\left\langle\boldsymbol{f}_{1}, \boldsymbol{l}_{1}\right\rangle^{2}} \boldsymbol{f}_{1}+\sum_{i=2}^{k} \boldsymbol{f}_{i}\left\langle\boldsymbol{f}_{i}, \boldsymbol{z}\right\rangle
$$

where $\left\{\boldsymbol{f}_{1}, \ldots, \boldsymbol{f}_{k}\right\}$ is the orthonormal with respect to $\langle\cdot, \cdot\rangle$ basis of $T_{\boldsymbol{c}} \boldsymbol{\Omega}, \boldsymbol{h}$ is orthogonal to $T,\langle\boldsymbol{h}, \boldsymbol{h}\rangle=1, \boldsymbol{H}_{\boldsymbol{c}}^{-1} \nabla G=\alpha \boldsymbol{f}_{1}+\beta \boldsymbol{h}, \boldsymbol{l}_{1}=\left(\beta \boldsymbol{f}_{1}-\alpha \boldsymbol{h}\right) / \sqrt{\alpha^{2}+\beta^{2}},\left\langle\boldsymbol{f}_{1}, \boldsymbol{l}_{1}\right\rangle=\beta / \sqrt{\alpha^{2}+\beta^{2}}$.

Thus, for solving the invariance equation iteratively, one needs only projector $\boldsymbol{P}_{\boldsymbol{c}}$ (83), and one does not need a priori parameterization of $\Omega$ anymore.

\section{Method of invariant grids}

Elsewhere above in this paper, we considered the invariant manifold, and methods for their construction, without addressing the question of how to implement it in a constructive way. In most of the works (of us and of other people on similar problems), analytic forms were needed to represent manifolds. However, in order to construct manifolds of a relatively low dimension, grid-based representations of manifolds become a relevant option. The Method of invariant grids (MIG) was suggested recently in [32].

The principal idea of (MIG) is to find a mapping of finite-dimensional grids into the phase space of a dynamic system. That is we construct not just a point approximation of the invariant manifold, but an invariant grid. When refined, in the limit it is expected to converge, of course, to the invariant manifold, but it is a separate, independently defined object.

Let's denote $L=\boldsymbol{R}^{n}, \mathbf{G}$ is a discrete subset of $\boldsymbol{R}^{n}$. A natural choice would be a regular grid, but, this is not crucial from the point of view of the general formalism. For every point $y \in \mathbf{G}$, a neighborhood of $y$ is defined: $V_{y} \subset \mathbf{G}$, where $V_{y}$ is a finite set, and, in particular, $y \in V_{y}$. On regular grids, $V_{y}$ includes, as a rule, the nearest neighbors of $y$. It may also include next to nearest points.

For our purposes, one should define a grid differential operator. For every function, defined on the grid, also all derivatives are defined:

$$
\left.\frac{\partial f}{\partial y_{i}}\right|_{y \in \mathbf{G}}=\sum_{z \in V_{y}} q_{i}(z, y) f(z), i=1, \ldots n .
$$


where $q_{i}(z, y)$ are some coefficients.

Here we do not specify the choice of the functions $q_{i}(z, y)$. We just mention in passing that, as a rule, equation (84) is established using some interpolation of $f$ in the neighborhood of $y$ in $\boldsymbol{R}^{n}$ by some differentiable functions (for example, polynomial). This interpolation is based on the values of $f$ in the points of $V_{y}$. For regular grids, $q_{i}(z, y)$ are functions of the difference $z-y$. For some $y$ s which are close to the edges of the grid, functions are defined only on the part of $V_{y}$. In this case, the coefficients in (84) should be modified appropriately in order to provide an approximation using available values of $f$. Below we will assume this modification is always done. We also assume that the number of points in the neighborhood $V_{y}$ is always sufficient to make the approximation possible. This assumption restricts the choice of the grids $\mathbf{G}$. Let's call admissible all such subsets $\mathbf{G}$, on which one can define differentiation operator in every point.

Let $F$ be a given mapping of some admissible subset $\mathbf{G} \subset \boldsymbol{R}^{n}$ into $U$. For every $y \in V$ we define tangent vectors:

$$
T_{y}=\operatorname{Lin}\left\{\boldsymbol{g}_{i}\right\}_{1}^{n}
$$

where vectors $\boldsymbol{g}_{i}(i=1, \ldots n)$ are partial derivatives $(84)$ of the vector-function $F$ :

$$
\boldsymbol{g}_{i}=\frac{\partial F}{\partial y_{i}}=\sum_{z \in V_{y}} q_{i}(z, y) F(z),
$$

or in the coordinate form:

$$
\left(\boldsymbol{g}_{i}\right)_{j}=\frac{\partial F_{j}}{\partial y_{i}}=\sum_{z \in V_{y}} q_{i}(z, y) F_{j}(z) .
$$

Here $\left(\boldsymbol{g}_{i}\right)_{j}$ is the $j$ th coordinate of the vector $\left(\boldsymbol{g}_{i}\right)$, and $F_{j}(z)$ is the $j$ th coordinate of the point $F(z)$.

The grid $\mathbf{G}$ is invariant, if for every node $y \in \mathbf{G}$ the vector field $J(F(y))$ belongs to the tangent space $T_{y}$ (here $J$ is the right hand site of the kinetic equations (4)).

So, the definition of the invariant grid includes:

1) Finite admissible subset $\mathbf{G} \subset \boldsymbol{R}^{n}$;

2) A mapping $F$ of this admissible subset $\mathbf{G}$ into $U$ (where $U$ is the phase space for kinetic equations (4));

3) The differentiation formulas (84) with given coefficients $q_{i}(z, y)$;

The grid invariance equation has a form of inclusion: 


$$
\boldsymbol{J}(F(y)) \in T_{y} \text { for every } y \in \mathbf{G}
$$

or a form of equation:

$$
\left(1-\boldsymbol{P}_{F(y)}\right) \boldsymbol{J}(F(y))=0 \text { for every } y \in \mathbf{G},
$$

where $\boldsymbol{P}_{F(y)}$ is the thermodynamic projector (83).

The grid differentiation formulas (84) are needed, in the first place, to establish the tangent space $T_{y}$, and the null space of the thermodynamic projector $\boldsymbol{P}_{F(y)}$ in each node. It is important to realise that locality of construction of thermodynamic projector enables this without a need for a global parametrization.

Basically, in our approach, the grid specifics are in: (a) differentiation formulas, (b) grid construction strategy (the grid can be extended, contracted, refined, etc.) The invariance equations (45), the iteration Newton method $(46,47)$, and the formulas of the relaxation approximation (77) do not change at all. For convenience, let us repeat all these formulas in the grid context.

Let $\boldsymbol{c}=F(y)$ be position of a grid's node $y$ immersed into phase space $U$. We have set of tangent vectors $\boldsymbol{g}_{i}(x)$, defined in $\boldsymbol{c}(86)$, (87). Thus, the tangent space $T_{y}$ is defined by (85). Also, one has the thermodynamic Lyapunov function $G(\boldsymbol{c})$, the linear functional $\left.D_{\boldsymbol{c}} G\right|_{\boldsymbol{c}}$, and the subspace $T_{0 y}=\left.T_{y} \cap \operatorname{ker} D_{\boldsymbol{c}} G\right|_{\boldsymbol{c}}$ in $T_{y}$. Let $T_{0 y} \neq T_{y}$. In this case we have a vector $\boldsymbol{e}_{y} \in T_{y}$, orthogonal to $T_{0 y},\left.D \boldsymbol{c} G\right|_{\boldsymbol{c}}\left(\boldsymbol{e}_{y}\right)=1$. Then, the thermodynamic projector is defined as:

$$
\boldsymbol{P}_{\boldsymbol{c}} \bullet=\boldsymbol{P}_{0 \boldsymbol{c}} \bullet+\left.\boldsymbol{e}_{y} D_{\boldsymbol{c}} G\right|_{\boldsymbol{c}} \bullet
$$

where $\boldsymbol{P}_{0 \boldsymbol{c}}$ is the orthogonal projector on $T_{0 y}$ with respect to the entropic scalar product $\langle,\rangle_{x}$.

If $T_{0 y}=T_{y}$, then the thermodynamic projector is the orthogonal projector on $T_{y}$ with respect to the entropic scalar product $\langle,\rangle \boldsymbol{c}$.

For the Newton method with incomplete linearization, the equations for calculating new node position $\boldsymbol{c}^{\prime}=\boldsymbol{c}+\delta \boldsymbol{c}$ are:

$$
\left\{\begin{array}{l}
\boldsymbol{P}_{\boldsymbol{c}} \delta \boldsymbol{c}=0 \\
\left(1-\boldsymbol{P}_{\boldsymbol{c}}\right)(\boldsymbol{J}(\boldsymbol{c})+D \boldsymbol{J}(\boldsymbol{c}) \delta \boldsymbol{c})=0 .
\end{array}\right.
$$

Here $D \boldsymbol{J}(\boldsymbol{c})$ is a matrix of derivatives of $\boldsymbol{J}$, calculated in $\boldsymbol{c}$. The self-adjoint linearization may be useful too (see section 5.2). 
Equation (89) is a system of linear algebraic equations. In practice, it is convenient to choose some orthonormal (with respect to the entropic scalar product) basis $\boldsymbol{b}_{i}$ in $k e r \boldsymbol{P}_{\boldsymbol{c}}$. Let $r=\operatorname{dim}\left(\operatorname{ker} \boldsymbol{P}_{\boldsymbol{c}}\right)$. Then $\delta \boldsymbol{c}=\sum_{i=1}^{r} \delta_{i} \boldsymbol{b}_{i}$, and the system looks like

$$
\sum_{k=1}^{r} \delta_{k}\left\langle\boldsymbol{b}_{\mathbf{i}}, D \boldsymbol{J}(\boldsymbol{c}) \boldsymbol{b}_{k}\right\rangle_{\boldsymbol{c}}=-\left\langle\boldsymbol{J}(\boldsymbol{c}), \boldsymbol{b}_{i}\right\rangle \boldsymbol{c}, i=1 \ldots r .
$$

Here $\langle,\rangle_{\boldsymbol{c}}$ is the entropic scalar product (28). This is the system of linear equations for adjusting the node position accordingly to the Newton method with incomplete linearization.

For the relaxation method, one needs to calculate the defect $\Delta_{\boldsymbol{c}}=\left(1-\boldsymbol{P}_{\boldsymbol{c}}\right) \boldsymbol{J}(\boldsymbol{c})$, and the relaxation step

$$
\tau(x)=-\frac{\left\langle\Delta_{\boldsymbol{c}}, \Delta_{\boldsymbol{c}}\right\rangle_{\boldsymbol{c}}}{\left\langle\Delta_{\boldsymbol{c}}, D \boldsymbol{J}(\boldsymbol{c}) \Delta_{\boldsymbol{c}}\right\rangle_{\boldsymbol{c}}}
$$

Then, new node position $x^{\prime}$ is calculated as

$$
\boldsymbol{c}^{\prime}=\boldsymbol{c}+\tau(\boldsymbol{c}) \Delta \boldsymbol{c}
$$

This is the equation for adjusting the node position according to the relaxation method.

\subsection{Grid construction strategy}

From all reasonable strategies of the invariant grid construction we will consider here the following two: growing lump and invariant flag.

\subsubsection{Growing lump}

In this strategy one chooses as initial the equilibrium point $y^{*}$. The first approximation is constructed as $F\left(y^{*}\right)=\boldsymbol{c}^{*}$, and for some initial $V_{0}\left(V_{y^{*}} \subset V_{0}\right)$ one has $F(y)=\boldsymbol{c}^{*}+A\left(y-y^{*}\right)$, where $A$ is an isometric embedding (in the standard Euclidean metrics) of $\boldsymbol{R}^{n}$ in $E$.

For this initial grid one makes a fixed number of iterations of one of the methods chosen (Newton's method with incomplete linearization or the relaxation method), and, after that, puts $V_{1}=\bigcup_{y \in V_{0}} V_{y}$ and extends $F$ from $V_{0}$ onto $V_{1}$ using linear extrapolation and the process continues. One of the possible variants of this procedure is to extend the grid from $V_{i}$ to $V_{i+1}$ not after a fixed number of iterations, but when the invariance defect $\Delta_{y}$ becomes smaller than a given $\epsilon$ (in a given norm, which is entropic, as a rule), for all nodes $y \in V_{i}$. The lump stops growing when it reaches the boundary and is within a given accuracy $\|\Delta\|<\epsilon$. 


\subsubsection{Invariant flag}

For the invariant flag one uses sufficiently regular grids $\mathbf{G}$, in which many points are situated on the coordinate lines, planes, etc. One considers the standard flag $\boldsymbol{R}^{0} \subset \boldsymbol{R}^{1} \subset$ $\boldsymbol{R}^{2} \subset \ldots \subset \boldsymbol{R}^{n}$ (every next space is constructed by adding one more coordinate). It corresponds to a succession of grids $\{y\} \subset \mathbf{G}^{1} \subset \mathbf{G}^{2} \ldots \subset \mathbf{G}^{n}$, where $\left\{y^{*}\right\}=\boldsymbol{R}^{0}$, and $\mathbf{G}^{i}$ is a grid in $\boldsymbol{R}^{i}$.

First, $y^{*}$ is mapped in $c^{*}$ and further $F\left(y^{*}\right)=\boldsymbol{c}^{*}$. Then an invariant grid is constructed on $V^{1} \subset \mathbf{G}^{1}$ (up to the boundaries $U$ and within a given accuracy $\|\Delta\|<\epsilon$ ). After the neighborhoods in $\mathbf{G}^{2}$ are added to the points $V^{1}$, and, using such extensions, the grid $V^{2} \subset \mathrm{G}^{2}$ is constructed (up to the boundaries and within a given accuracy) and so on, until $V^{n} \subset \mathrm{G}^{n}$ will be constructed.

We must underline here that, constructing the k-th grid $V^{k} \subset \mathrm{G}^{k}$, the important role of the grids of smaller dimension $V^{0} \subset \ldots \subset V^{k-1} \subset V^{k}$ embedded in it, is preserved. The point $F\left(y^{*}\right)=x^{*}$ is preserved. For every $y \in V^{q}(q<k)$ the tangent vectors $g_{1}, \ldots, g_{q}$ are constructed, using the differentiation operators (84) on the whole $V^{k}$. Using the tangent space $T_{y}=\operatorname{Lin}\left\{g_{1}, . ., g_{q}\right\}$, the projector $\boldsymbol{P}_{F(y)}$ is constructed, the iterations are applied and so on. All this is done to obtain a succession of embedded invariant grids, given by the same map $F$.

\subsubsection{Boundaries check and the entropy}

We construct grid mapping of $F$ onto the finite set $V \in \mathbf{G}$. The technique of checking if the grid still belongs to the phase space $U$ of kinetic system $(F(V) \subset U)$ is quite straightforward: all the points $y \in V$ are checked to belong to $U$. If at the next iteration a point $F(y)$ leaves $U$, then it is returned inside by a homothety transform with the center in $x^{*}$. Since the thermodynamic Lyapunov function is a convex function, the homothety contraction with the center in $x^{*}$ decreases it monotonously. Another variant is cutting off the points leaving $U$.

By the way it was constructed (83) the kernel of the thermodynamic projector is annulled by the entropy differential. Thus, in the first order, steps in the Newton method with incomplete linearization $(46,47)$ as well as in the relaxation methods $(76),(77)$ do not change the entropy. But, if the steps are quite large, then the increasing of the thermodynamic Lyapunov function can become essential and the points are returned on their level by the homothety contraction with the center in the equilibrium point.

\subsection{Instability of fine grids}

When one reduces the grid step (spacing between the nodes) in order to get a finer grid, then, starting from a definite step, it is possible to face the problem of the Courant instability [16]. Instead of converging, at the every iteration the grid becomes entangled 
(see Fig. 2).

The way to get rid off this instability is well-known. This is decreasing the time step. Instead of the real time step, we have a shift in the Newtonian direction. Formally, we can assign for one complete step in the Newtonian direction a value $h=1$. Let us consider now the Newton method with an arbitrary $h$. For this, let us find $\delta \boldsymbol{c}=\delta F(y)$ from (89), but we will change $\delta \boldsymbol{c}$ proportionally to $h$ : the new value of $\boldsymbol{c}_{n+1}=F_{n+1}(y)$ will be equal to

$$
F_{n+1}(y)=F_{n}(y)+h_{n} \delta F_{n}(y)
$$

where the lower index $n$ denotes the step number.

One way to choose the $h$ step value is to make it adaptive, controlling the average value of the invariance defect $\left\|\Delta_{y}\right\|$ at every step. Another way is the convergence control: then $\sum h_{n}$ plays a role of time.

Elimination of Courant instability for the relaxation method can be made quite analogously. Everywhere the step $h$ is maintained as big as it is possible without convergence problems.

\subsection{What space is the most appropriate for the grid construction?}

For the kinetics systems there are two distinguished representations of the phase space:

- The densities space (concentrations, energy or probability densities, etc.)

- The spaces of conjugate intensive quantities, potentials (temperature, chemical potentials, etc.)

The density space is convenient for the construction of quasi-chemical representations. Here the balance relations are linear and the restrictions are in the form of linear inequalities (the densities themselves or some linear combinations of them must be positive).

The conjugate variables space is convenient in the sense that the equilibrium conditions, given the linear restrictions on the densities, are in the linear form (with respect to the conjugate variables). In these spaces the quasi-equilibrium manifolds exist in the form of linear subspaces and, vise versa, linear balance equations turns out to be equations of the conditional entropy maximum.

The duality we've just described is very well-known and studied in details in many works on thermodynamics and Legendre transformations [13,73]. In the previous section, the grids were constructed in the density space. But the procedure of constructing them in the space of the conjugate variables seems to be more consistent. The principal argument for this is the specific role of quasi-equilibrium, which exists as a linear manifold. Therefore, linear extrapolation gives a thermodynamically justified quasi-equilibrium ap- 
proximation. Linear approximation of the slow invariant manifold in the neighborhood of the equilibrium in the conjugate variables space already gives the global quasi-equilibrium manifold, which corresponds to the motion separation (for slow and fast motions) in the neighborhood of the equilibrium point.

For the mass action law, transition to the conjugate variables is simply the logarithmic transformation of the coordinates.

10.4 Carleman's formulas in the analytical invariant manifolds approximations. First profit from analyticity: superresolution

When constructing invariant grids, one must define the differential operators (84) for every grid node. For calculating the differential operators in some point $y$, an interpolation procedure in the neighborhood of $y$ is used. As a rule, it is an interpolation by a loworder polynomial, which is constructed using the function values in the nodes belonging to the neighbourhood of $y$ in $\mathbf{G}$. This approximation (using values in the closest nodes) is natural for smooth functions. But, for the systems (4) with analytical right hand side we are looking for the analytical invariant manifold (due to Lyapunov auxiliary theorem $[60,54])$. Analytical functions have much more "rigid" structure than the smooth ones. One can change a smooth function in the neighborhood of any point in such a way, that outside this neighborhood the function will not change. In general, this is not possible for analytical functions: a kind of "long-range" effect takes place (as is well known) .

The idea is to use this effect and to reconstruct some analytical function $f_{\mathbf{G}}$ using function given on $\mathbf{G}$. There is one important requirement: if these values on $\mathbf{G}$ are values (given in the points of $\mathbf{G}$ ) of some function $f$ which is analytical in the given neighborhood $U$, then if the $\mathbf{G}$ is refined "correctly", one must have $f_{\mathbf{G}} \rightarrow f$. The sequence of reconstructed function $f_{\mathbf{G}}$ should should converge to the "proper" function $f$.

What is the "correct refinement"? For smooth functions for the convergence $f_{\mathbf{G}} \rightarrow f$ it is necessary and sufficient that, in the course of refinement, G would approximate the whole $U$ with arbitrary accuracy. For analytical functions it is only necessary that, under the refinement, $\mathbf{G}$ would approximate some uniqueness set ${ }^{3} A \subset U$. Suppose we have a sequence of grids $\mathbf{G}$, each next is finer than previous, which approximates a set $A$. For smooth functions, using function values defined on the grids, one can reconstruct the function in $A$. For analytical functions, if the analyticity area $U$ is known, and $A$ is a uniqueness set in $U$, then one can reconstruct the function in $U$. The set $U$ can be essentially bigger than $A$; because of this such extension was named as superresolution effects $[1,38]$. There exist constructive formulas for construction of analytical functions $f_{\mathbf{G}}$ for different areas $U$, uniqueness sets $A \subset U$ and for different ways of discrete approximation of $A$ by a sequence of fined grids $\mathbf{G}$ [1]. Here we provide only one Carleman's formula which is the most appropriate for our purposes.

$\overline{3 \text { Let's }}$ remind to the reader that $A \subset U$ is called uniqueness set in $U$ if for analytical in $U$ functions $\psi$ and $\varphi$ from $\left.\left.\psi\right|_{A} \equiv \varphi\right|_{A}$ it follows $\psi=\varphi$. 
Let area $U=Q_{\sigma}^{n} \subset C^{n}$ be a product of strips $Q_{\sigma} \subset C, Q_{\sigma}=\{z \mid \operatorname{Im} z<\sigma\}$. We will construct functions holomorphic in $Q_{\sigma}^{n}$. This is effectively equivalent to the construction of real analytical functions $f$ in whole $\boldsymbol{R}^{n}$ with a condition on the convergence radius $r(x)$ of the Taylor series for $f$ as a function of each coordinate: $r(x) \geq \sigma$ in every point $x \in \boldsymbol{R}^{n}$.

The sequence of fined grids is constructed as follows: let for every $l=1, \ldots, n$ a finite sequence of distinct points $N_{l} \subset D_{\sigma}$ be defined:

$$
N_{l}=\left\{x_{l j} \mid j=1,2,3 \ldots\right\}, x_{l j} \neq x_{l i} \text { for } i \neq j
$$

The uniqueness set $A$, which is approximated by a sequence of fined finite grids, has the form:

$$
A=N_{1} \times N_{2} \times \ldots \times N_{n}=\left\{\left(x_{1 i_{1}}, x_{2 i_{2}}, . ., x_{n i_{n}}\right) \mid i_{1, . ., n}=1,2,3, \ldots\right\}
$$

The grid $\mathbf{G}_{m}$ is defined as the product of initial fragments $N_{l}$ of length $m$ :

$$
\mathbf{G}_{m}=\left\{\left(x_{1 i_{1}}, x_{2 i_{2}} \ldots x_{n i_{n}}\right) \mid 1 \leq i_{1, . ., n} \leq m\right\}
$$

Let's denote $\lambda=2 \sigma / \pi$ ( $\sigma$ is a half-width of the strip $Q_{\sigma}$ ). The key role in the construction of the Carleman's formula is played by the functional $\omega_{m}^{\lambda}(u, p, l)$ of 3 variables: $u \in U=$ $Q_{\sigma}^{n}, p$ is an integer, $1 \leq p \leq m, l$ is an integer, $1 \leq p \leq n$. Further $u$ will be the coordinate value in the point where the extrapolation is calculated, $l$ will be the coordinate number, and $p$ will be an element of multi-index $\left\{i_{1}, \ldots, i_{n}\right\}$ for the point $\left(x_{1 i_{1}}, x_{2 i_{2}}, \ldots, x_{n i_{n}}\right) \in \mathbf{G}$ :

$$
\omega_{m}^{\lambda}(u, p, l)=\frac{\left(e^{\lambda x_{l p}}+e^{\lambda \bar{x}_{l p}}\right)\left(e^{\lambda u}-e^{\lambda x_{l p}}\right)}{\lambda\left(e^{\lambda u}+e^{\lambda \bar{x}_{l p}}\right)\left(u-x_{l p}\right) e^{\lambda x_{l p}}} \times \prod_{j=1 j \neq p}^{m} \frac{\left(e^{\lambda x_{l p}}+e^{\lambda \bar{x}_{l j}}\right)\left(e^{\lambda u}-e^{\lambda x_{l j}}\right)}{\left(e^{\lambda x_{l p}}-e^{\lambda x_{l j}}\right)\left(e^{\lambda u}+e^{\lambda \bar{x}_{l j}}\right)}
$$

For real-valued $x_{p k}$ formula (97) becomes simpler:

$$
\omega_{m}^{\lambda}(u, p, l)=2 \frac{e^{\lambda u}-e^{\lambda x_{l p}}}{\lambda\left(e^{\lambda u}+e^{\lambda x_{l p}}\right)\left(u-x_{l p}\right)} \times \prod_{j=1 j \neq p}^{m} \frac{\left(e^{\lambda x_{l p}}+e^{\lambda x_{l j}}\right)\left(e^{\lambda u}-e^{\lambda x_{l j}}\right)}{\left(e^{\lambda x_{l p}}-e^{\lambda x_{l j}}\right)\left(e^{\lambda u}+e^{\lambda x_{l j}}\right)}
$$

The Carleman's formula for extrapolation from $\mathbf{G}_{M}$ on $U=Q_{\sigma}^{n}(\sigma=\pi \lambda / 2)$ has the form $\left(z=\left(z_{1}, \ldots, z_{n}\right)\right)$ :

$$
f_{m}(z)=\sum_{k_{1}, \ldots, k_{n}=1}^{m} f\left(x_{k}\right) \prod_{j=1}^{n} \omega_{m}^{\lambda}\left(z_{j}, k_{j}, j\right)
$$

where $k=k_{1}, . ., k_{n}, x_{k}=\left(x_{1 k_{1}}, x_{2 k_{2}}, \ldots, x_{n k_{n}}\right)$.

There exists a theorem [1]: 
If $f \in H^{2}\left(Q_{\sigma}^{n}\right)$, then $f(z)=\lim _{m \rightarrow \infty} f_{m}(z)$, where $H^{2}\left(Q_{\sigma}^{n}\right)$ is the Hardy class of holomorphic in $Q_{\sigma}^{n}$ functions.

It is useful to present the asymptotics of (99) for big $\left|\operatorname{Re} z_{j}\right|$. For this we will consider the asymptotics of (99) for big $|\operatorname{Reu}|$ :

$$
\left|\omega_{m}^{\lambda}(u, p, l)\right|=\left|\frac{2}{\lambda u} \prod_{j=1 j \neq p}^{m} \frac{e^{\lambda x_{l p}}+e^{\lambda x_{l j}}}{e^{\lambda x_{l p}}-e^{\lambda x_{l j}}}\right|+o\left(|\operatorname{Re} u|^{-1}\right) .
$$

From the formula (99) one can see that for the finite $m$ and $\left|\operatorname{Re} z_{j}\right| \rightarrow \infty$ function $\left|f_{m}(z)\right|$ behaves like const $\cdot \prod_{j}\left|z_{j}\right|^{-1}$.

This property (null asymptotics) must be taken into account when using the formula (99). When constructing invariant manifolds $F(W)$, it is natural to use (99) not for the immersion $F(y)$, but for the deviation of $F(y)$ from some analytical ansatz $F_{0}(y)$.

The analytical ansatz $F_{0}(y)$ can be obtained using Taylor series, just as in the Lyapunov auxiliary theorem [60]. Another variant is using Taylor series for the construction of Padeapproximations.

It is natural to use approximations (99) in dual variables as well, since there exists for them (as the examples demonstrate) a simple and very effective linear ansatz for the invariant manifold. This is the slow invariant subspace $E_{\text {slow }}$ of the operator of linearized system (4) in dual variables in the equilibrium point. This invariant subspace corresponds to the the set of "slow" eigenvalues (with small $|\operatorname{Re} \lambda|, \operatorname{Re} \lambda<0$ ). In the initial space (of concentrations or densities) this invariant subspace is the quasi-equilibrium manifold. It consist of the maximal entropy points on the affine manifolds of the $x+E_{\text {fast }}$ form, where $E_{\text {fast }}$ is the "fast" invariant subspace of the operator of linearized system (4) in the initial variables in the equilibrium point. It corresponds to the "fast" eigenvalues (big $|\operatorname{Re} \lambda|$, $\operatorname{Re} \lambda<0)$.

\subsection{Example: Two-step catalytic reaction}

Let's consider a two-step four-component reaction with one catalyst $A_{2}$ :

$$
A_{1}+A_{2} \leftrightarrow A_{3} \leftrightarrow A_{2}+A_{4}
$$

We assume the Lyapunov function of the form $\mathbf{G}=\sum_{i=1}^{4} c_{i}\left[\ln \left(c_{i} / c_{i}^{*}\right)-1\right]$. The kinetic equation for the four-component vector of concentrations, $\mathbf{c}=\left(c_{1}, c_{2}, c_{3}, c_{4}\right)$, has the form

$$
\dot{\mathbf{c}}=\gamma_{1} W_{1}+\gamma_{2} W_{2} .
$$


Here $\gamma_{1,2}$ are stoichiometric vectors,

$$
\gamma_{1}=(-1,-1,1,0), \gamma_{2}=(0,1,-1,1)
$$

while functions $W_{1,2}$ are reaction rates:

$$
W_{1}=k_{1}^{+} c_{1} c_{2}-k_{1}^{-} c_{3}, W_{2}=k_{2}^{+} c_{3}-k_{2}^{-} c_{2} c_{4}
$$

Here $k_{1,2}^{ \pm}$are reaction rate constants. The system under consideration has two conservation laws,

$$
c_{1}+c_{3}+c_{4}=B_{1}, c_{2}+c_{3}=B_{2},
$$

or $\left\langle\boldsymbol{b}_{1,2}, \boldsymbol{c}\right\rangle=B_{1,2}$, where $\boldsymbol{b}_{1}=(1,0,1,1)$ and $\boldsymbol{b}_{1}=(0,1,1,0)$. The nonlinear system (101) is effectively two-dimensional, and we consider a one-dimensional reduced description. For our example, we chose the following set of parameters:

$$
\begin{aligned}
& k_{1}^{+}=0.3, k_{1}^{-}=0.15, k_{2}^{+}=0.8, k_{2}^{-}=2.0 \\
& c_{1}^{*}=0.5, c_{2}^{*}=0.1, c_{3}^{*}=0.1, c_{4}^{*}=0.4 \\
& B_{1}=1.0, B_{2}=0.2
\end{aligned}
$$

In Fig. 3 one-dimensional invariant grid is shown in the $\left(c_{1}, c_{4}, c_{3}\right)$ coordinates. The grid was constructed by growing the grid, as described above. We used Newtonian iterations to adjust the nodes. The grid was grown up to the boundaries of the phase space.

The grid derivatives for calculating tangent vectors $\boldsymbol{g}$ were taken as simple as $\boldsymbol{g}\left(\boldsymbol{c}_{i}\right)=$ $\left(\boldsymbol{c}_{i+1}-\boldsymbol{c}_{i-1}\right) /\left\|\boldsymbol{c}_{i+1}-\boldsymbol{c}_{i-1}\right\|$ for the internal nodes and $\boldsymbol{g}\left(\boldsymbol{c}_{1}\right)=\left(\boldsymbol{c}_{1}-\boldsymbol{c}_{2}\right) /\left\|\boldsymbol{c}_{1}-\boldsymbol{c}_{2}\right\|, \boldsymbol{g}\left(\boldsymbol{c}_{n}\right)=$ $\left(\boldsymbol{c}_{n}-\boldsymbol{c}_{n-1}\right) /\left\|\boldsymbol{c}_{n}-\boldsymbol{c}_{n-1}\right\|$ for the grid's boundaries. Here $x_{i}$ denotes the vector of the $i$ th node position, $n$ is the number of nodes in the grid.

Close to the phase space boundaries we had to apply an adaptive algorithm for choosing the time step $h$ : if, after the next growing step and applying $N=20$ complete Newtonian steps, the grid did not converged, then we choose a new $h_{n+1}=h_{n} / 2$ and recalculate the grid. The final value for $h$ was $h \approx 0.001$.

The nodes positions are parametrized with entropic distance to the equilibrium point measured in the quadratic metrics given by $\boldsymbol{H}_{\boldsymbol{c}}=\left\|\partial^{2} G(\boldsymbol{c}) / \partial c_{i} \partial c_{j}\right\|$ in the equilibrium $\boldsymbol{c}^{*}$. It means that every node is on a sphere in this quadratic metrics with a given radius, which increases linearly. On this figure the step of the increase is chosen to be 0.05 . Thus, the first node is on the distance 0.05 from the equilibrium, the second is on the distance 0.10 and so on. Fig. 4 shows several basic values which facilitate understanding of the object (invariant grid) extracted. The sign on the x-axis of the graphs at Fig. 4 is meaningless, since the distance is always positive, but in this situation it denotes two possible directions from the equilibrium point. 
Fig. 4a,b effectively represents the slow one-dimensional component of the dynamics of the system. Given any initial condition, the system quickly finds the corresponding point on the manifold and starting from this point the dynamics is given by a part of the graph on the Fig. 4a,b.

One of the useful values is shown on the Fig. 4c. It is the relation between the relaxation times "toward" and "along" the grid $\left(\lambda_{2} / \lambda_{1}\right.$, where $\lambda_{1}, \lambda_{2}$ are the smallest and the second smallest by absolute value non-zero eigenvalue of the system, symmetrically linearized in the point of the grid node). It shows that the system is very stiff close to the equilibrium point, and less stiff (by one order of magnitude) on the borders. This leads to the conclusion that the reduced model is more adequate in the neighborhood of the equilibrium where fast and slow motions are separated by two orders of magnitude. On the very end of the grid which corresponds to the positive absciss values, our one-dimensional consideration faces with definite problems (slow manifold is not well-defined).

\subsection{Example: Model hydrogen burning reaction}

In this section we consider a more interesting illustration, where the phase space is 6dimensional, and the system is 4-dimensional. We construct an invariant flag which consists of 1- and 2-dimensional invariant manifolds.

We consider chemical system with six species called (provisionally) $\mathrm{H}_{2}$ (hydrogen), $\mathrm{O}_{2}$ (oxygen), $\mathrm{H}_{2} \mathrm{O}$ (water), $\mathrm{H}, \mathrm{O}, \mathrm{OH}$ (radicals). We assume the Lyapunov function of the form $G=\sum_{i=1}^{6} c_{i}\left[\ln \left(c_{i} / c_{i}^{*}\right)-1\right]$. The subset of the hydrogen burning reaction and corresponding (direct) rate constants have been taken as:

$$
\begin{array}{ll}
\text { 1. } \mathrm{H}_{2} \leftrightarrow 2 H & k_{1}^{+}=2 \\
\text { 2. } \mathrm{O}_{2} \leftrightarrow 2 O & k_{2}^{+}=1 \\
\text { 3. } \mathrm{H}_{2} O \leftrightarrow H+O H & k_{3}^{+}=1 \\
\text { 4. } \mathrm{H}_{2}+\mathrm{O} \leftrightarrow H+O H & k_{4}^{+}=10^{3} \\
\text { 5. } \mathrm{O}_{2}+\mathrm{H} \leftrightarrow \mathrm{O}+\mathrm{OH} & k_{5}^{+}=10^{3} \\
\text { 6. } \mathrm{H}_{2}+\mathrm{O} \leftrightarrow \mathrm{H}_{2} \mathrm{O} & k_{6}^{+}=10^{2}
\end{array}
$$

The conservation laws are:

$$
\begin{aligned}
& 2 c_{\mathrm{H}_{2}}+2 c_{\mathrm{H}_{2} \mathrm{O}}+c_{H}+c_{\mathrm{O}} H=b_{H} \\
& 2 c_{\mathrm{O}_{2}}+c_{\mathrm{H} 2 \mathrm{O}}+c_{O}+c_{O} H=b_{O}
\end{aligned}
$$

For parameter values we took $b_{H}=2, b_{O}=1$, and the equilibrium point:

$$
c_{H_{2}}^{*}=0.27 c_{O_{2}}^{*}=0.135 c_{H_{2} O}^{*}=0.7 c_{H}^{*}=0.05 c_{O}^{*}=0.02 c_{O H}^{*}=0.01
$$


Other rate constants $k_{i}^{-}, i=1 . .6$ were calculated from $\boldsymbol{c}^{*}$ value and $k_{i}^{+}$. For this system the stoichiometric vectors are:

$$
\begin{aligned}
\gamma_{1} & =(-1,0,0,2,0,0) \quad \gamma_{2}=(0,-1,0,0,2,0) \\
\gamma_{3} & =(0,0,-1,1,0,1) \quad \gamma_{4}=(-1,0,0,1,-1,1) \\
\gamma_{5} & =(0,-1,0,-1,1,1) \quad \gamma_{6}=(-1,0,1,0,-1,0)
\end{aligned}
$$

We stress here once again that the system under consideration is fictional in that sense that the subset of equations corresponds to the simplified picture of this physical-chemical process and the constants do not correspond to any measured ones, but reflect only basic orders of magnitudes of the real-world system. In this sense we consider here a qualitative model system, which allows us to illustrate the invariant grids method without excessive complication. Nevertheless, modeling of real systems differs only in the number of species and equations. This leads, of course, to computationally harder problems, but not the crucial ones, and the efforts on the modeling of real-world systems are on the way.

Fig. 5a presents a one-dimensional invariant grid constructed for the system. Fig. 5b shows the picture of reduced dynamics along the manifold (for the explanation of the meaning of the $x$-coordinate, see the previous subsection). On Fig. $5 \mathrm{c}$ the three smallest by absolute value non-zero eigen values of the symmetrically linearized system $A^{\text {sym }}$ have been shown. One can see that the two smallest values "exchange" on one of the grid end. It means that one-dimensional "slow" manifold has definite problems in this region, it is just not defined there. In practice, it means that one has to use at least two-dimensional grids there.

Fig. 6a gives a view onto the two-dimensional invariant grid, constructed for the system, using the "invariant flag" strategy. The grid was grown starting from the 1D-grid constructed at the previous step. At the first iteration for every node of the initial grid, two nodes (and two edges) were added. The direction of the step was chosen as the direction of the eigenvector of the matrix $A^{\text {sym }}$ (in the point of the node), corresponding to the second "slowest" direction. The value of the step was chosen to be $\epsilon=0.05$ in terms of entropic distance. After several Newtonian iterations done until convergence, new nodes were added in the direction "ortogonal" to the 1D-grid. This time it is done by linear extrapolation of the grid on the same step $\epsilon=0.05$. When some new nodes have one or several negative coordinates (the grid reaches the boundaries) they were cut off. If a new node has only one edge, connecting it to the grid, it was excluded (since it does not allow calculating 2D-tangent space for this node). The process continues until the expansion is possible (after this, every new node has to be cut off).

Strategy of calculating tangent vectors for this regular rectangular 2D-grid was chosen to be quite simple. The grid consists of rows, which are co-oriented by construction to the initial 1D-grid, and columns that consist of the adjacent nodes in the neighboring rows. The direction of "columns" corresponds to the second slowest direction along the grid. Then, every row and column is considered as 1D-grid, and the corresponding tangent 
vectors are calculated as it was described before:

$$
\boldsymbol{g}_{\text {row }}\left(\boldsymbol{c}_{k, i}\right)=\left(\boldsymbol{c}_{k, i+1}-\boldsymbol{c}_{k, i-1}\right) /\left\|\boldsymbol{c}_{k, i+1}-\boldsymbol{c}_{k, i-1}\right\|
$$

for the internal nodes and

$$
\boldsymbol{g}_{\text {row }}\left(\boldsymbol{c}_{k, 1}\right)=\left(\boldsymbol{c}_{k, 1}-\boldsymbol{c}_{k, 2}\right) /\left\|\boldsymbol{c}_{k, 1}-\boldsymbol{c}_{k, 2}\right\|, \boldsymbol{g}_{\text {row }}\left(\boldsymbol{c}_{k, n_{k}}\right)=\left(\boldsymbol{c}_{k, n_{k}}-\boldsymbol{c}_{k, n_{k}-1}\right) /\left\|\boldsymbol{c}_{k, n_{k}}-\boldsymbol{c}_{k, n_{k}-1}\right\|
$$

for the nodes which are close to the grid's edges. Here $x_{k, i}$ denotes the vector of the node in the $k$ th row, $i$ th column; $n_{k}$ is the number of nodes in the $k$ th row. Second tangent vector $\boldsymbol{g}_{\text {col }}\left(\boldsymbol{c}_{k, i}\right)$ is calculated completely analogously. In practice, it is convenient to orthogonalize $\boldsymbol{g}_{\text {row }}\left(\boldsymbol{c}_{k, i}\right)$ and $g_{\text {col }}\left(\boldsymbol{c}_{k, i}\right)$.

Since the phase space is four-dimensional, it is impossible to visualize the grid in one of the coordinate 3D-views, as it was done in the previous subsection. To facilitate visualization one can utilize traditional methods of multi-dimensional data visualization. Here we make use of the principal components analysis (see, for example, [45]), which constructs a threedimensional linear subspace with maximal dispersion of the othogonally projected data (grid nodes in our case). In other words, method of principal components constructs in multi-dimensional space such a three-dimensional box inside which the grid can be placed maximally tightly (in the mean square distance meaning). After projection of the grid nodes into this space, we get more or less adequate representation of the two-dimensional grid embedded into the six-dimensional concentrations space (Fig. 6b). The disadvantage of the approach is that the axes now do not have explicit meaning, being some linear combinations of the concentrations.

One attractive feature of two-dimensional grids is the possibility to use them as a screen, on which one can display different functions $f(\boldsymbol{c})$ defined in the concentrations space. This technology was exploited widely in the non-linear data analysis by the elastic maps method [41]. The idea is to "unfold" the grid on a plane (to present it in the two-dimensional space, where the nodes form a regular lattice). In other words, we are going to work in the internal coordinates of the grid. In our case, the first internal coordinate (let's call it $s_{1}$ ) corresponds to the direction, co-oriented with the one-dimensional invariant grid, the second one (let's call it $s_{2}$ ) corresponds to the second slow direction. By how it was constructed, $s_{2}=0$ line corresponds to the one-dimensional invariant grid. Units of $s_{1}$ and $s_{2}$ are entropic distances in our case.

Every grid node has two internal coordinates $\left(s_{1}, s_{2}\right)$ and, simultaneously, corresponds to a vector in the concentration space. This allows us to map any function $f(\mathbf{c})$ from the multidimensional concentration space to the two-dimensional space of the grid. This mapping is defined in a finite number of points (grid nodes), and can be interpolated (linearly, in the simplest case) in between them. Using coloring and isolines one can visualize the values of the function in the neighborhood of the invariant manifold. This is meaningful, since, by the definition, the system spends most of the time in the vicinity of the invariant manifold, thus, one can visualize the behaviour of the system. As a result of applying the technology, one obtains a set of color illustrations (a stack of information layers), put onto the grid as a map. This allows applying all the methods, working with stack of 
information layers, like geographical information systems (GIS) methods, which are very well developed.

In short words, the technique is a useful tool for exploration of dynamical systems. It allows to see simultaneously many different scenarios of the system behaviour, together with different system's characteristics.

The simplest functions to visualize are the coordinates: $c_{i}(\boldsymbol{c})=c_{i}$. On Fig. 7 we displayed four colorings, corresponding to the four arbitrarily chosen concentrations functions (of $\mathrm{H}_{2}, \mathrm{O}, \mathrm{H}$ and $\mathrm{OH}$; Fig. 7a-d). The qualitative conclusions that can be made from the graphs are that, for example, the concentration of $H_{2}$ practically does not change during the first fast motion (towards the 1D-grid) and then, gradually changes to the equilibrium value (the $\mathrm{H}_{2}$ coordinate is "slow"). The $O$ coordinate is the opposite case, it is "fast" coordinate which changes quickly (on the first stage of motion) to the almost equilibrium value, and then it almost does not change. Basically, the slope angles of the coordinate isolines give some presentation of how "slow" a given concentration is. Fig. 7c shows interesting behaviour of the $O H$ concentration. Close to the $1 \mathrm{D}$ grid it behaves like "slow coordinate", but there is a region on the map where it has clear "fast" behaviour (middle bottom of the graph).

The next two functions which one can want to visualize are the entropy $S=-G$ and the entropy production $\sigma(\boldsymbol{c})=-d G / d t(\boldsymbol{c})=-\sum_{i} \ln \left(c_{i} / c_{i}^{*}\right) \dot{c}_{i}$. They are shown on Fig. 8a,b.

Finally, we visualize the relation between the relaxation times of the fast motion towards the 2D-grid and along it. This is given on the Fig. 8c. This picture allows to make a conclusion that two-dimensional consideration can be appropriate for the system (especially in the "high $\mathrm{H}_{2}$, high $O$ " region), since the relaxation times "towards" and "along" the grid are definitely separated. One can compare this to the Fig. 8d, where the relation between relaxation times towards and along the 1D-grid is shown.

\section{Method of invariant manifold for open systems}

One of the problems to be focused on when studying closed systems is to prepare extensions of the result for open or driven by flows systems. External flows are usually taken into account by additional terms in the kinetic equations (4):

$$
\dot{\boldsymbol{c}}=\boldsymbol{J}(\boldsymbol{c})+\Pi
$$

Zero-order approximation assumes that the flow does not change the invariant manifold. Equations of the reduced dynamics, however, do change: Instead of $\boldsymbol{J}(\boldsymbol{c}(M))$ we substitute $\boldsymbol{J}(\boldsymbol{c}(M))+\boldsymbol{\Pi}$ into Eq. (39):

$$
\dot{M}_{i}=\left(\left.\nabla M_{i}\right|_{\boldsymbol{c}(M)}, \boldsymbol{J}(\boldsymbol{c}(M))+\boldsymbol{\Pi}\right) .
$$


Zero-order approximation assumes that the fast dynamics in the closed system strongly couples the variables $\boldsymbol{c}$, so that flows cannot influence this coupling.

First-order approximation takes into account the shift of the invariant manifold by $\delta \boldsymbol{c}$. Equations for Newton's iterations have the same form (57) but instead of the vector field $\boldsymbol{J}$ they take into account the presence of the flow:

$$
\left[1-\boldsymbol{P}_{\boldsymbol{c}}\right]\left(\boldsymbol{\Pi}+\boldsymbol{L}_{\boldsymbol{c}}^{\prime} \delta \boldsymbol{c}\right)=0, \boldsymbol{P}_{\boldsymbol{c}} \delta \boldsymbol{c}=0
$$

where projector $\boldsymbol{P}_{\boldsymbol{c}}$ corresponds to the unperturbed manifold.

The first-order approximation means that fluxes change the coupling between the variables (concentrations). It is assumed that these new coupling is also set instantaneously (neglect of inertia).

Remark. Various realizations of the first-order approximation in physical and chemical dynamics implement the viewpoint of an infinitely small chemical reactor driven by the flow. In other words, this approximation is applicable in the Lagrangian system of coordinates $[51,79]$. Transition to Eulerian coordinates is possible but the relations between concentrations and the flow will change its form. In a contrast, the more simplistic zero-order approximation is equally applicable in both the coordinate system, if it is valid.

\section{Conclusion}

In this paper, we have presented the method for constructing the invariant manifolds for reducing systems of chemical kinetics. Our approach to computations of invariant manifolds of dissipative systems is close in spirit to the Kolmogorov-Arnold-Moser theory of invariant tori of Hamiltonian systems [5,6]: We also base our consideration on the Newton method instead of Taylor series expansions [7], and systematically use duality structures. Recently, a version of an approach based on the invariance equations was rediscovered in [54]. He was solving the invariance equation by a Taylor series expansion. A counterpart of Taylor series expansions for constructing the slow invariant manifolds in the classical kinetic theory is the famous Chapman-Enskog method. The question of how this compares to iteration methods was studied extensively for certain classes of Grad moment equations $[31,50,48]$.

The thermodynamic parameterization and the selfadjoint linearization arise in a natural way in the problem of finding slowest invariant manifolds for closed systems. This also leads to various applications in different approaches to reducing the description, in particular, to a thermodynamically consistent version of the intrinsic low-dimensional manifold, and to model kinetic equations for lifting the reduced dynamics. Use of the thermodynamic projector makes it unnecessary global parameterizations of manifolds, and thus leads to computationally promising grid-based realizations. 
Invariant manifolds are constructed for closed space-independent chemical systems. We also describe how to use these manifolds for modeling open and distributed systems.

Acknowledgements. We thank Dr. Vladimir Zmievski (Ecole Polytechnique Montreal) for computations in section 7. Fruitful discussions with Prof. Hans Christian Öttinger (ETH Zurich) on the problem of reduction are gratefully acknowledged. Encouragement of Prof. Misha Gromov (IHES Bures-sur-Yvette) was very important for completing this work.

\section{References}

[1] Aizenberg, L., Carleman's Formulas in Complex Analysis: Theory and Applications. (Mathematics and Its Applications; V. 244) Kluwer, 1993.

[2] Ansumali, S., \& Karlin, I. V. Stabilization of the Lattice Boltzmann method by the $H$ theorem: A numerical test. Phys. Rev. E, 62(6) (2000), 7999-8003.

[3] Ansumali S., \& Karlin, I. V. Single relaxation time model for entropic Lattice Boltzmann methods. Phys. Rev. E, 65 (2002), 056312(1-9).

[4] Ansumali, S., \& Karlin, I.V. Entropy function approach to the lattice Boltzmann method. J. Stat. Phys., 107(1/2) (2002), 291-308.

[5] Arnold, V. I. Proof of a theorem of A. N. Kolmogorov on the invariance of quasi-periodic motions under small perturbations of the Hamiltonian. (English translation). Russian Mathematical Surveys, 18 (1963), 9-36.

[6] Arnold, V. I. Geometrical methods in the theory of ordinary differential equations. New York: Springer, 1983.

[7] Beyn, W.-J., \& Kless, W. Numerical Taylor expansions of invariant manifolds in large dynamical systems. Numerische Mathematik, 80 (1998), 1-38.

[8] Bhatnagar, P. L., Gross, E. P., \& Krook, M. A model for collision processes in gases. Small amplitude processes in charged and neutral one-component systems. Phys. Rev., 94 (1954), 511-525.

[9] Bobylev, A. V., The theory of the nonlinear spatially uniform Boltzmann equation for Maxwell molecules. Mathematical physics reviews, 7 (1988), 111-233.

[10] Bowen, J. R., Acrivos, A., \& Oppenheim, A. K., Singular Perturbation Refinement to Quasi-Steady State Approximation in Chemical Kinetics, Chemical Engineering Science, 18 (1963), 177-188.

[11] Bykov, V. I., Gorban, A. N., \& Yablonskii, G. S. Description of nonisothermal reactions in terms of Marcelin-de Donder kinetics and its generalizations. React. Kinet. Catal. Lett., 20 (1982), 261-265.

[12] Bykov, V. I., Yablonskii, G. S., \& Akramov, T. A., The rate of the free energy decrease in the course of the complex chemical reaction. Dokl. Akad. Nauk USSR, 234 (3) (1977), 621-634. 
[13] Callen, H. B. Thermodynamics and an Introduction to Thermostatistics, (Wiley, New York), 1985.

[14] Chen, J.-Y., A general procedure for constructing reduced reaction mechanisms with given independent relations. Combustion Science and Technology, 57 (1988), 89-94.

[15] Chicone, C., \& Swanson, R., Linearization via the Lie derivative. Electron. J. Diff. Eqns., Monograph, 02 (2000), http://ejde.math.swt.edu or http://ejde.math.unt.edu ftp ejde.math.swt.edu or ejde.math.unt.edu (login: ftp)

[16] Courant, R., Friedrichs, K. O. and Lewy, H. On the partial difference equations of mathematical physics. IBM Journal, 215-234, March 1967. Ames, W.F., Numerical Methods for Partial Differential Equations, 2nd ed. (New York: Academic Press), 1977. Richtmyer, R.D., and Morton, K.W., Difference Methods for Initial Value Problems, 2nd ed. (New York: Wiley-Interscience), 1967.

[17] Dimitrov, V.I., Prostaya kinetika [Simple Kinetics]. Novosibirsk: Nauka. 1982.

[18] De Donder, T., \& Van Rysselberghe, P. Thermodynamic theory of affnity. A book of principles. Stanford: University Press, 1936.

[19] Dukek, G., Karlin, I. V., \& Nonnenmacher, T. F., Dissipative brackets as a tool for kinetic modeling. Physica A, 239(4) (1997), 493-508.

[20] Feinberg, M. On chemical kinetics of a certain class. Arch. Rational Mech. Anal., 46(1) (1972), 1-41.

[21] Fraser, S. J., The steady state and equilibrium approximations: A geometrical picture. J. Chem. Phys., 88(8) (1988), 4732-4738.

[22] Gear, C. W., Numerical initial value problems in ordinary differential equations. PrenticeHall, Englewood Cliffs, NJ, 1971.

[23] Gorban, A.N., Obkhod ravnovesiya [Equilibrium encircling]. Novosibirsk: Nauka, 1984.

[24] Gorban, A. N., \& Karlin, I. V. Thermodynamic parameterization. Physica A, 190 (1992), 393-404 .

[25] Gorban, A. N., \& Karlin, I. V. The constructing of invariant manifolds for the Boltzmann equation, Adv. Model. and Analysis C, 33(3) (1992), 39-54.

[26] Gorban, A. N., \& Karlin, I. V. Coarse-grained quasi- equilibrium approximations for kinetic equations. Adv. Model. and Analysis C, 35(1) (1992), 17-27.

[27] Gorban, A. N., \& Karlin, I. V. H-theorem for generalized models of the Boltzmann equation. Adv. Model. and Analysis C, 33(3) (1992), 33-38.

[28] Gorban, A. N., \& Karlin, I. V., Method of invariant manifolds and regularization of acoustic spectra. Transport Theory and Stat. Phys., 23 (1994), 559-632.

[29] Gorban, A. N., \& Karlin, I. V., General approach to constructing models of the Boltzmann equation. Physica A, 206 (1994), 401-420.

[30] Gorban, A. N., \& Karlin, I. V., Scattering rates versus moments: Alternative Grad equations. Phys. Rev. E, 54(4) (1996), R3109-R3113. 
[31] Gorban, A. N., \& Karlin, I. V., Short-wave limit of hydrodynamics: A soluble example. Phys. Rev. Lett., 77 (1996), 282-285.

[32] Gorban, A. N., Karlin, I. V., Method of invariant manifold for chemical kinetics, Chem. Eng. Sci., to appear. Preprint online: http://arxiv.org/abs/cond-mat/0207231, 9 Jul 2002.

[33] Gorban, A. N., Karlin, I. V., Zmievskii, V. B., \& Nonnenmacher, T. F. Relaxational trajectories: global approximations. Physica A, 231 (1996), 648-672.

[34] Gorban, A. N., Karlin, I. V., \& Zmievskii, V. B., Two-step approximation of spaceindependent relaxation. Transp. Theory Stat. Phys., 28(3) (1999), 271-296.

[35] Gorban, A. N., Karlin, I. V., Zmievskii, V. B., \& Dymova S. V., Reduced description in reaction kinetics. Physica A, 275(3-4) (2000), 361-379.

[36] Gorban, A. N., Karlin, I. V., Ilg, P., \& Öttinger, H. C., Corrections and enhancements of quasi-equilibrium states. J. Non- Newtonian Fluid Mech., 96(1-2) (2001), 203-219.

[37] Gorban, A. N., Pitenko, A. A., Zinovyev, A. Y., \& Wunsch, D.C., Vizualization of any data using elastic map method. Smart Engineering System Design, 11 (2001), 363-368.

[38] Gorban, A. N., \& Rossiev, A. A. Neural network iterative method of principal curves for data with gaps. Journal of Computer and System Sciences International, 38(5) (1999), $825-831$.

[39] Gorban A., Rossiev A., Makarenko N., Kuandykov Y., Dergachev V. Recovering data gaps through neural network methods. International Journal of Geomagnetism and Aeronomy, 3, No. 2 (2002), 191-197.

[40] Gorban, A. N., Rossiev, A. A., Wunsch, D. C. II, Neural network modeling of data with gaps: method of principal curves, Carleman's formula, and other, The talk was given at the USA-NIS Neurocomputing opportunities workshop, Washington DC, July 1999 (Associated with IJCNN'99). Online: http://arXiv.org/abs/cond-mat/0305508, 21 May 2003.

[41] Gorban, A. N., Zinovyev, A. Yu., Visualization of data by method of elastic maps and its applications in genomics, economics and sociology. Institut des Hautes Etudes Scientifiques, Preprint (2001), IHES M/01/36. Online: http://www.ihes.fr/PREPRINTS/M01/Resu/resu-M01-36.html.

[42] Grmela, M., Karlin, I. V., \& Zmievski, V. B., Boundary layer minimum entropy principles: A case study. Phys. Rev. E, 66 (2002), 011201.

[43] Hartman, P., On the local linearization of differential equations. Proc. Amer. Math. Soc., 14 (1963), 568-573.

[44] Hartman, P., Ordinary differential equations. Boston: Birkhäuser, 1982.

[45] Jolliffe, I. T. Principal Component Analysis. Springer-Verlag, 1986.

[46] Karlin, I. V., On the relaxation of the chemical reaction rate. In: Mathematical Problems of Chemical Kinetics, eds. K. I. Zamaraev and G. S. Yablonskii, (Nauka, Novosibirsk) (1989), $7-42$.

[47] Karlin, I. V., The problem of reduced description in kinetic theory of chemically reacting gas. Modeling, Measurement and Control C, 34(4) (1993), 1-34. 
[48] Karlin, I. V. Exact summation of the Chapman-Enskog expansion from moment equations. J. Phys. A: Math. Gen., 33 (2000), 8037-8046.

[49] Karlin, I. V., Dukek, G., \& Nonnenmacher, T. F. Invariance principle for extension of hydrodynamics: Nonlinear viscosity. Phys. Rev. E, 55(2) (1997), 1573-1576.

[50] Karlin, I. V., Dukek, G., \& Nonnenmacher, T. F. Gradient expansions in kinetic theory of phonons. Phys. Rev. B, 55 (1997), 6324-6329.

[51] Karlin, I. V., Gorban, A. N., Dukek, \& G., Nonnenmacher, T. F., Dynamic correction to moment approximations. Phys. Rev. E, 57 (1998), 1668-1672.

[52] Karlin, I. V., \& Zmievskii, V. B., Invariant closure for the Fokker-Planck equation, 1998. Preprint online: http://arxiv.org/abs/adap-org/9801004 4 Feb 1998.

[53] Kato, T., Perturbation theory for Linear operators. Berlin: Springer, 1976.

[54] Kazantzis N., Singular PDEs and the problem of finding invariant manifolds for nonlinear dynamical systems. Physics Letters, A272(4) (2000), 257-263.

[55] Khibnik, A., Kuznetsov, Y., Levitin, V., \& Nikolaev, E., Continuation techniques and interactive software for bifurcation analysis of ODEs and iterated maps. Physica D, 62 (1993), 360-370.

[56] Lam, S.H., Goussis, D. A., The CSP Method for Simplifying Kinetics. International Journal of Chemical Kinetics, 26 (1994), 461-486.

[57] Levenspiel, O., Chemical Reaction Engineering. Ind. Eng. Chem. Res., 38 (1999), 4139-4143.

[58] Levenspiel, O., Response to Professor Yablonsky. Ind. Eng. Chem. Res., 39 (2000), 3120.

[59] Li, G., Rabitz, H., \& Tóth, J., A general analysis of exact nonlinear lumping in chemical kinetics. Chem. Eng. Sci., 49(3) (1994), 343-361.

[60] Lyapunov A.M. The general Problem of the Stability of Motion, Taylor \& Francis, 1992.

[61] Maas, U., \& Pope, S.B., Simplifying chemical kinetics: intrinsic low- dimensional manifolds in composition space. Combustion and Flame, 88 (1992), 239-264.

[62] Orlov, N. N., \& Rozonoer, L. I., The macrodynamics of open systems and the variational principle of the local potential. J. Franklin Inst., 318 (1984), 283-314 and 315-347.

[63] Rabitz, H., Chemical Dynamics and Kinetics Phenomena as revealed by Sensitivity Analysis Techniques. Chem. Rev., 87 (1987), 101-112.

[64] Rabitz, H., Kramer, M., \& Dacol, D., Sensitivity analysis in chemical kinetics. Ann. Rev. Phys. Chem., 34 (1983), 419-461.

[65] Roose, De Dier, B., \& Spence, A., eds., Continuation and bifurcations numerical techniques and applications. Dordrecht: Kluwer, 1990.

[66] Roussel, M.R., \& Fraser, S.J., Geometry of the steady-state approximation: Perturbation and accelerated convergence methods. J. Chem. Phys. (1990), 93, 1072-1081.

[67] Roussel, M.R., \& Fraser, S.J., On the geometry of transient relaxation. J. Chem. Phys., 94 (1991), 7106-711. 
[68] Segel, L.A., \& Slemrod, M., The quasi-steady-state assumption: A case study in perturbation. SIAM Rev., 31 (1989), 446-477.

[69] Struchtrup, H., \& Weiss, W., Maximum of the local entropy production becomes minimal in stationary processes. Phys. Rev. Lett., 80 (1998), 5048-5051.

[70] Strygin V. V., \& Sobolev, V. A., Splitting of motion by means of integral manifolds. Moscow: Nauka, 1988.

[71] Tóth, J., Li, G., Rabitz, H., \& Tomlin, A. S., The effect of lumping and expanding on kinetic differential equations. SIAM J. Appl. Math., 57(6) (1997), 1531-1556.

[72] Vasil'eva A.B., Butuzov V.F., \& Kalachev L.V., The boundary function method for singular perturbation problems, Philadelphia: SIAM, 1995.

[73] Use of Legendre Transforms in Chemical Thermodynamics (IUPAC Technical Report), Prepared for publication by R. A. Alberty. Pure Appl.Chem., Vol.73, No.8 (2001), pp.13491380. Online: www.iupac.org/publications/pac/2001/pdf/7308x1349.pdf

[74] Volpert, A. I., \& Hudjaev, S. I., Analysis in classes of discontinuous functions and the equations of mathematical physics. Dordrecht: Nijhoff, 1985.

[75] Wei, J., \& Prater, C., The structure and analysis of complex reaction systems. Adv. Catalysis, 13 (1962), 203-393.

[76] Yablonsky, G. S., Comments on a commentary by Professor Levenspiel, Ind. Eng. Chem. Res., 39 (2000), 3120.

[77] Yablonskii, G.S., Bykov, V.I., Gorban, A.N., \& Elokhin, V.I., Kinetic models of catalytic reactions. Comprehensive Chemical Kinetics, Vol. 32, Compton, R. G., ed. Amsterdam: Elsevier, 1991.

[78] Zhu, W., \& Petzold, L., Model reduction for chemical kinetics: An optimization approach. AIChE Journal, (April 1999), 869-886.

[79] Zmievskii, V. B., Karlin, I. V., \& Deville, M., The universal limit in dynamics of dilute polymeric solutions. Physica A, 275(1-2) (2000), 152-177. 
Fig. 1. Images of the initial quasi-equilibrium manifold (bold line) and the first two corrections (solid normal lines) in the phase plane $\left[c_{1}, c_{3}\right]$ for two-step catalytic reaction. Dashed lines are individual trajectories.

Fig. 2. Grid instability. For small grid steps approximations in the calculation of grid derivatives lead to the grid instability effect. On the figure several successive iterations of the algorithm without adaptation of the time step are shown that lead to undesirable "oscillations", which eventually destruct the grid starting from one of it's ends.

Fig. 3. One-dimensional invariant grid (circles) for two-dimensional chemical system. Projection into the $3 \mathrm{~d}$-space of $c_{1}, c_{4}, c_{3}$ concentrations. The trajectories of the system in the phase space are shown by lines. The equilibrium point is marked by square. The system quickly reaches the grid and further moves along it.

Fig. 4. One-dimensional invariant grid for two-dimensional chemical system. a) Values of the concentrations along the grid. b) Values of the entropy $(-G)$ and the entropy production $(-d G / d t)$ along the grid. c) Relation of the relaxation times "toward" and "along" the manifold. The nodes positions are parametrized with entropic distance measured in the quadratic metrics given by $\mathbf{H}_{\mathbf{c}}=\left\|\partial^{2} G(\mathbf{c}) / \partial c_{i} \partial c_{j}\right\|$ in the equilibrium $c^{*}$. Zero corresponds to the equilibrium.

Fig. 5. One-dimensional invariant grid for model hydrogen burning system. a) Projection into the 3 d-space of $c_{H}, c_{O}, c_{O H}$ concentrations. b) Concentration values along the grid. c) three smallest by absolute value non-zero eigen values of the symmetrically linearized system.

Fig. 6. Two-dimensional invariant grid for the model hydrogen burning system. a) Projection into the $3 \mathrm{~d}$-space of $c_{H}, c_{O}, c_{O H}$ concentrations. b) Projection into the principal 3D-subspace. Trajectories of the system are shown coming out from the every grid node. Bold line denotes the one-dimensional invariant grid, starting from which the $2 \mathrm{D}$-grid was constructed.
a) Concentration $\mathrm{H}_{2}$
b) Concentration $O$
c) Concentration $\mathrm{OH}$
d) Concentration $H$

Fig. 7. Two-dimensional invariant grid as a screen for visualizing different functions defined in the concentrations space. The coordinate axes are entropic distances (see the text for the explanations) along the first and the second slowest directions on the grid. The corresponding $1 \mathrm{D}$ invariant grid is denoted by bold line, the equilibrium is denoted by square.
a) Entropy
c) $\lambda_{3} / \lambda_{2}$ relation
b) Entropy Production
d) $\lambda_{2} / \lambda_{1}$ relation

Fig. 8. Two-dimensional invariant grid as a screen for visualizing different functions defined in the concentrations space. The coordinate axes are entropic distances (see the text for the explanations) along the first and the second slowest directions on the grid. The corresponding $1 \mathrm{D}$ invariant grid is denoted by bold line, the equilibrium is denoted by square. 
This figure "fig1.jpg" is available in "jpg" format from: http://arxiv.org/ps/cond-mat/0307076v1 
This figure "fig2.jpg" is available in "jpg" format from: http://arxiv.org/ps/cond-mat/0307076v1 
This figure "fig3.jpg" is available in "jpg" format from: http://arxiv.org/ps/cond-mat/0307076v1 
This figure "fig4.jpg" is available in "jpg" format from: http://arxiv.org/ps/cond-mat/0307076v1 
This figure "fig5.jpg" is available in "jpg" format from: http://arxiv.org/ps/cond-mat/0307076v1 
This figure "fig6.jpg" is available in "jpg" format from: http://arxiv.org/ps/cond-mat/0307076v1 
This figure "fig7.jpg" is available in "jpg" format from: http://arxiv.org/ps/cond-mat/0307076v1 
This figure "fig8.jpg" is available in "jpg" format from: http://arxiv.org/ps/cond-mat/0307076v1 